ARTICLE

DOI: $10.1038 / \mathrm{s} 41467-018-02856-2$

\title{
Characterizing steroid hormone receptor chromatin binding landscapes in male and female breast cancer
}

Tesa M. Severson ${ }^{1}$, Yongsoo Kim¹,2, Stacey E.P. Joosten², Karianne Schuurman², Petra van der Groep ${ }^{3}$, Cathy B. Moelans ${ }^{3}$, Natalie D. ter Hoeve ${ }^{3}$, Quirine F. Manson ${ }^{3}$, John W. Martens (D) 4,5,6, Carolien H.M. van Deurzen ${ }^{4,5}$, Ellis Barbe ${ }^{4,7}$, Ingrid Hedenfalk ${ }^{8}$, Peter Bult ${ }^{9}$, Vincent T.H.B.M. Smit ${ }^{4,10}$, Sabine C. Linn $3,11,12$, Paul J. van Diest ${ }^{3}$, Lodewyk Wessels ${ }^{1,6,13} \&$ Wilbert Zwart $^{2}$

Male breast cancer $(\mathrm{MBC})$ is rare and poorly characterized. Like the female counterpart, most $M B C s$ are hormonally driven, but relapse after hormonal treatment is also noted. The panhormonal action of steroid hormonal receptors, including estrogen receptor alpha (ER $\alpha)$, androgen receptor (AR), progesterone receptor (PR), and glucocorticoid receptor $(G R)$ in this understudied tumor type remains wholly unexamined. This study reveals genomic cross-talk of steroid hormone receptor action and interplay in human tumors, here in the context of $M B C$, in relation to the female disease and patient outcome. Here we report the characterization of human breast tumors of both genders for cistromic make-up of hormonal regulation in human tumors, revealing genome-wide chromatin binding landscapes of ER $\alpha, A R, P R, G R$, FOXA1, and GATA3 and enhancer-enriched histone mark H3K4me1. We integrate these data with transcriptomics to reveal gender-selective and genomic location-specific hormone receptor actions, which associate with survival in $\mathrm{MBC}$ patients.

\footnotetext{
${ }^{1}$ Division of Molecular Carcinogenesis, Oncode Institute, Netherlands Cancer Institute, 1066 CX Amsterdam, The Netherlands. ${ }^{2}$ Division of Oncogenomics, Oncode Institute, Netherlands Cancer Institute, 1066 CX Amsterdam, The Netherlands. ${ }^{3}$ Department of Pathology, University Medical Center, 3584 CX Utrecht, The Netherlands. ${ }^{4}$ Dutch Breast Cancer Research Group, (BOOG Study Center), 1076 CV Amsterdam, The Netherlands. ${ }^{5}$ Department of Medical Oncology, Erasmus Medical Center, 3015 CE Rotterdam, The Netherlands. ${ }^{6}$ Cancer Genomics Netherlands, Universiteitsweg 100, 3584 CG Utrecht, The Netherlands. ${ }^{7}$ Department of Pathology, VU Medical Center (VUMC), 1081 HV Amsterdam, The Netherlands. ${ }^{8}$ Division of Oncology and Pathology, Lund University, 22100 Lund, Sweden. ${ }^{9}$ Department of Pathology, Radboud University Medical Center, 6525 GA Nijmegen, The Netherlands. ${ }^{10}$ Department of Pathology, Leiden University Medical Centre, 2333 ZA Leiden, The Netherlands. ${ }^{11}$ Division of Medical Oncology, Netherlands Cancer Institute, Amsterdam, 1066 CX, The Netherlands. ${ }^{12}$ Division of Molecular Pathology, Netherlands Cancer Institute, Amsterdam, 1066 CX, The Netherlands. ${ }^{13}$ Faculty of EEMCS, Delft University of Technology, 2628 CD Delft, The Netherlands. Tesa M. Severson, Yongsoo Kim and Stacey E.P. Joosten contributed equally to this work. Correspondence and requests for materials should be addressed to W.Z. (email: w.zwart@nki.nl)
} 
B reast cancer in men is rare and largely understudied. Male breast cancer (MBC) accounts for around 1\% of all 1.67 million breast cancers diagnosed each year, worldwide ${ }^{1}$. As compared to the female counterpart, $\mathrm{MBC}$ is a distinct disease regarding clinicopathological features (e.g., age of onset and frequency of hormone receptor positivity ${ }^{2}$ ) and molecular features (e.g., frequency of $B R C A 2$ mutation $^{3}$ and gene expression subtypes $^{4}$ ).

The majority of male (and female) breast cancers are hormonally driven ${ }^{5}$, where $\mathrm{ER} \alpha$ genomic action dictates transcriptional programs that drive tumor cell proliferation ${ }^{6}$. Analogous to the female counterpart, male breast cancers are treated with endocrine therapies (such as tamoxifen) to block ER $\alpha$ transcriptional activity, yet relapse after hormonal treatment has also been noted $^{2,7}$. Even though the genomic action of ER $\alpha$ in MBC remains completely elusive, multiple reports have studied ER $\alpha$ genomics in the female disease. ER $\alpha$-DNA binding profiles in tumors are dynamically affected by endocrine therapeutics ${ }^{8}$ and can differentiate female patients on outcome ${ }^{9,10}$. Cell line studies revealed ER $\alpha$-DNA binding and ER $\alpha$-driven transcriptional activation and cell proliferation to depend on its pioneer factors, including FOXA $1^{11}$ and GATA $3^{12}$.

Apart from $\mathrm{ER} \alpha$, other steroid hormone receptors are expressed in breast cancer as well, including androgen receptor $(A R)^{13}$, progesterone receptor $(\mathrm{PR})^{14}$, and glucocorticoid receptor $(\mathrm{GR})^{14}$. AR expression is frequently observed in most male (and female) breast cancers ${ }^{13-16}$, although its role in breast cancer is poorly understood $^{17}$. AR activation in breast cancer cells facilitates downstream gene expression that drives tumorigenesis in a similar manner to $\mathrm{ER} \alpha^{16}$. This tumorigenic action of AR is most extensively studied in prostate cancer ${ }^{18,19}$, where differential ARDNA binding profiles can classify prostate cancer patients on outcome ou-22 $^{20}$.

$\mathrm{AR}$ and $\mathrm{PR}$ are favorable prognostic markers in female breast cancer $(\mathrm{FBC})^{23,24}$. In addition, PR has recently been shown to modulate ER $\alpha$-DNA binding, directly reprogramming ER $\alpha$-driven transcriptional programs ${ }^{25}$. GR expression has been associated with FOXA1 and GATA3 expression in ER $\alpha$-positive FBC, and is associated with a favorable outcome in this patient population ${ }^{26}$. Its functional role in breast cancer in relation to other steroid hormone receptors is poorly characterized. Cumulatively, these data illuminate the likely interplay between different steroid hormone receptors in breast cancer. Although ER $\alpha$ cistromics has previously been studied in female breast tumors $^{9,10}$, and its interplay with transcription factors has been reported in cell lines ${ }^{10,11,15,27-31}$, all these transcription factors have never been profiled together in a single study in human breast tumors.

We have characterized DNA binding of six different hormonerelated transcription factors in an understudied field of human pathophysiology: male breast cancer. Through multidimensional genomic data integration on the level of transcription factor binding, copy number cistrome profiling (using off-target sequencing reads from ChIP-seq data) ${ }^{32}$, transcriptomics and the enhancer enriched histone mark H3K4me1, we present a first comprehensive overview of male breast cancer, which we compared with the female counterpart. This comprehensive overview reveals gender-selective and genomic location-specific hormone receptor action, which associate with survival in $\mathrm{MBC}$.

\section{Results}

Steroid hormone receptor profiling in male breast cancer. We aimed to generate a compendium of (epi)genomic, transcriptomic and clinical data for $49 \mathrm{ER} \alpha$-positive MBC samples to better characterize the molecular makeup of this disease. To determine the chromatin binding landscape of $\mathrm{ER} \alpha$ in relation to steroid hormone receptors $\mathrm{AR}, \mathrm{PR}$, and $\mathrm{GR}$ and its pioneer factors FOXA1 and GATA3, we performed ChIP-seq analyses in clinical specimens from patients who did not receive any therapy prior to surgery. These results were integrated with gene expression data and compared with female breast cancer and cell line ChIP-seq data (Fig. 1a, Supplementary Fig. 1). Samples were selected randomly for ChIP-seq of different factors. In this pioneering work, each transcription factor was profiled in $\mathrm{MBC}$ for the first time (30 ER $\alpha$ ChIP-seq datastreams and $\geq 7$ samples/factor for other factors with the exception of GATA3 and PR, with 3 and 4 samples, respectively). To position these results into epigenetic context, H3K4mel was included as active enhancer marker ${ }^{33}$. We generated RNA-seq data for the series, used to classify samples on subtypes related to outcome; M1 (poor) and M2 (good) ${ }^{4}$ (Supplementary Fig. 1). Finally, we used copy number data (detected using off-target sequencing reads) ${ }^{32}$ and RNA-seq data to perform integrative clustering (IntClust) classification, which was previously associated with FBC prognostication (Supplementary Fig. 1$)^{34}$. As expected, IntClust classifications and intrinsic subtypes (based on immunohistochemistry) were enriched for ER $\alpha$ positive classifications (29/30 and 28/28, respectively). Clinical data, such as number of positive lymph nodes at diagnosis and survival status were included for male (Supplementary Fig. 1 and Supplementary Table 1) and female patients (Supplementary Table 2). Missing clinical data are indicated in Supplementary Table 1. We identified bound regions (peaks) for each factor: ER $\alpha$ (biological replicate in Supplementary Fig. 2), FOXA1, AR, GR, PR, and GATA3 (ChIP-seq validated by ChIP-QPCR in Supplementary Fig. 3) using validated antibodies (Supplementary Fig. 4A, B), shown at two well-known ER $\alpha$ bound regions in FBC: loci RARA and GREB1 (Fig. 1b). In this principal examination of SHRs, FOXA1 and GATA3 in a tumor series, we observed these two ER $\alpha$-regions were bound by all other factors. This is reminiscent of FBC transcription factors, such as GATA3, FOXA1, RAR $\alpha$, which have been found to be bound in the same regions ${ }^{35}$. These findings were confirmed on a genome-wide scale, where all $\mathrm{ER} \alpha$ sites were considered bound in $\geq 50 \%$ of tumors in which we find co-binding by all other factors tested (Fig. 1c). In accordance with reports in cell lines ${ }^{11,25,29,36}$, all factors studied mainly bind intronic and distal intergenic regions (Fig. 1d). DNA motif analysis revealed self-preference for all factors (Fig. 1e, Supplementary Fig. 5) except for GATA3 as was reported previously ${ }^{12}$.

Interplay of ER $\alpha$ with other SHRs and pioneer factors. We have shown ER $\alpha$ binding sites have considerable overlap with other factors studied. Next, overlap of ER $\alpha$ with the other steroid hormone receptors (Fig. 2a, left) or pioneer factors FOXA1 and GATA3 (Fig. 2a, right) was studied. All sites shared for individual factors: ER $\alpha$ (15 out of 30 samples), AR (5 out of 10 samples), FOXA1 ( 3 out of 7 samples), PR ( 2 out of 4 samples), GR (3 out of 7 samples) and GATA3 (1 out of 3 samples). AR and GR have virtually no unique binding regions, while selective sites for $\mathrm{ER} \alpha$ and $\mathrm{PR}$ are identified. When examining ER $\alpha$, FOXA1 and GATA3, we identified selective sites in each. Strikingly, $71 \%$ of FOXA1 sites were FOXA1-selective while ER and GATA3selective sites were $42 \%$ and $33 \%$, respectively. Next, we counted the reads in the union of sites for all factors (Fig. 2a) and computed the correlation score for each sample (Pearson correlation coefficient), which was represented in a correlation matrix (Fig. 2b). Contrary to what is described for ER $\alpha / F O X A 1$ behavior in FBC cell lines ${ }^{11}$, we observed FOXA1 profiles do not correlate with other profiles, while all SHRs and GATA3 cluster together. Most notably, a strong similarity in genomic profiles of ER $\alpha$ with GR and AR was found. Practically all AR sites were co-occupied 
a

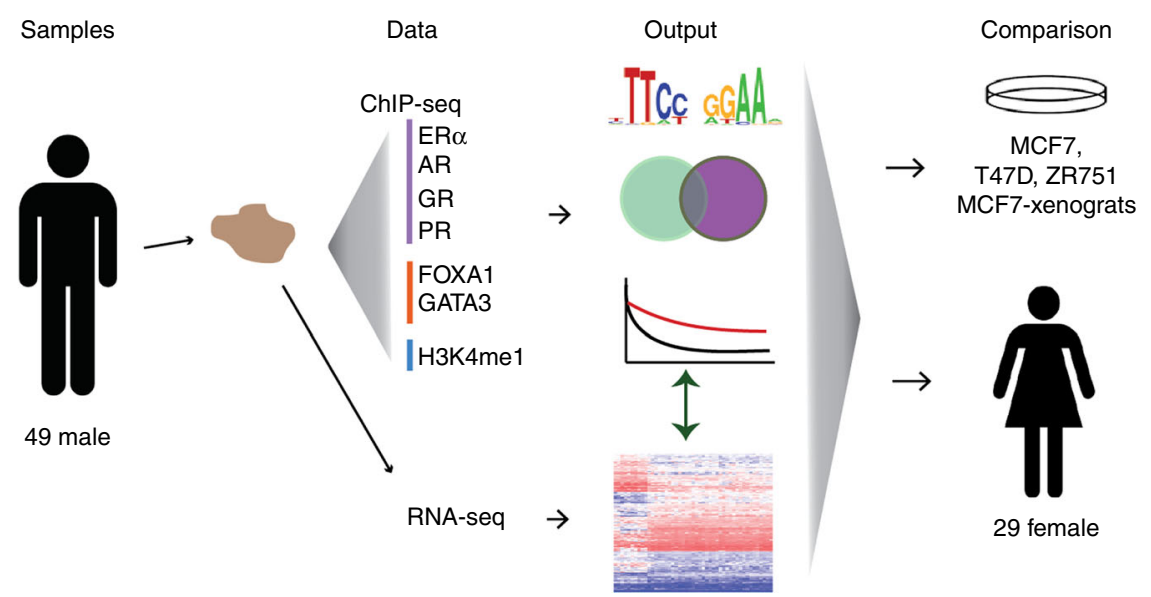

b

ChIP-seq snapshots

chr2:11,677,536-11,682,518 chr17:38,477,090-38,480,558

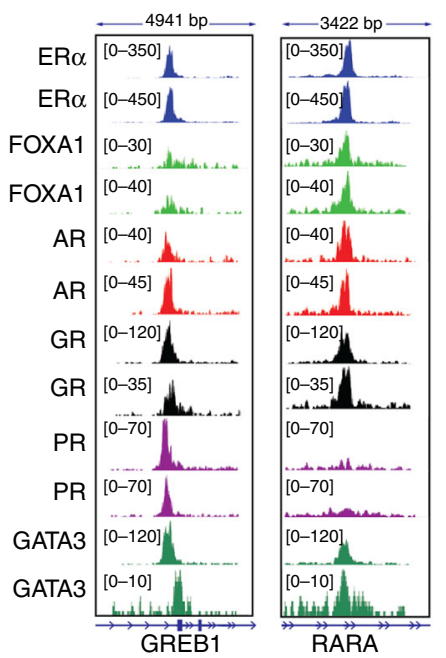

C ChIP-seq of different factors at ER $\alpha$ binding sites

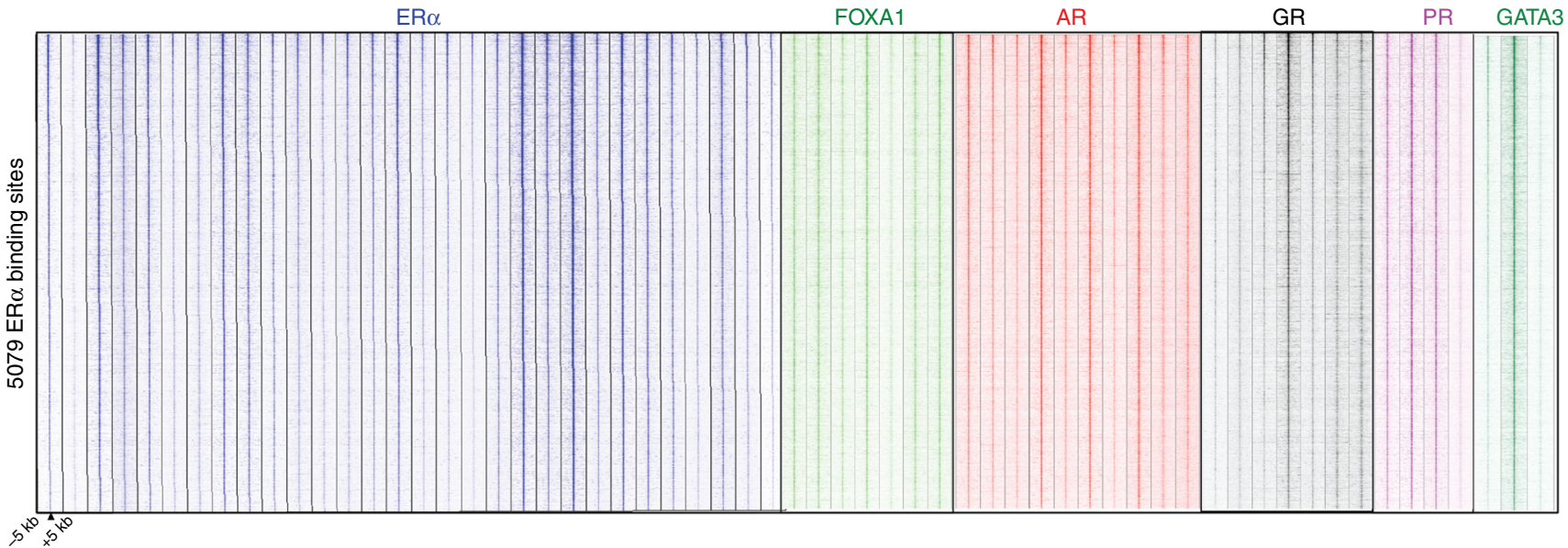

d Genomic distribution of binding regions for different factors
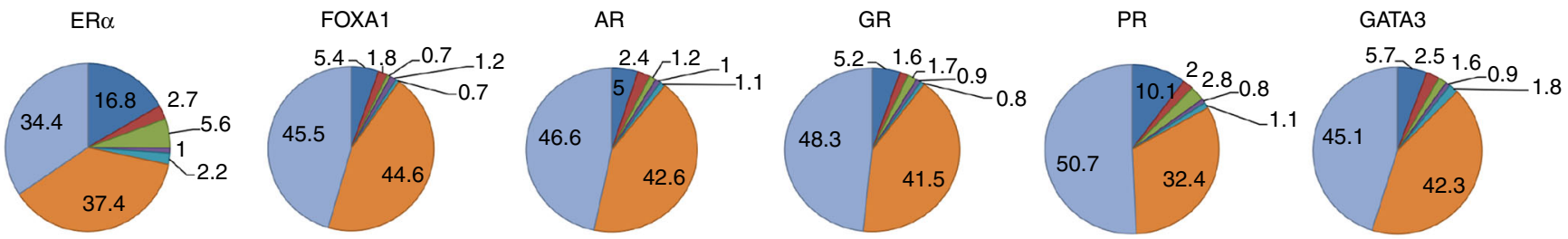

Promoter

Downstream $5^{\prime}$ UTR

3' UTR —Coding exon —Intron Distal integenic

e Sequence motifs of binding regions for different factors
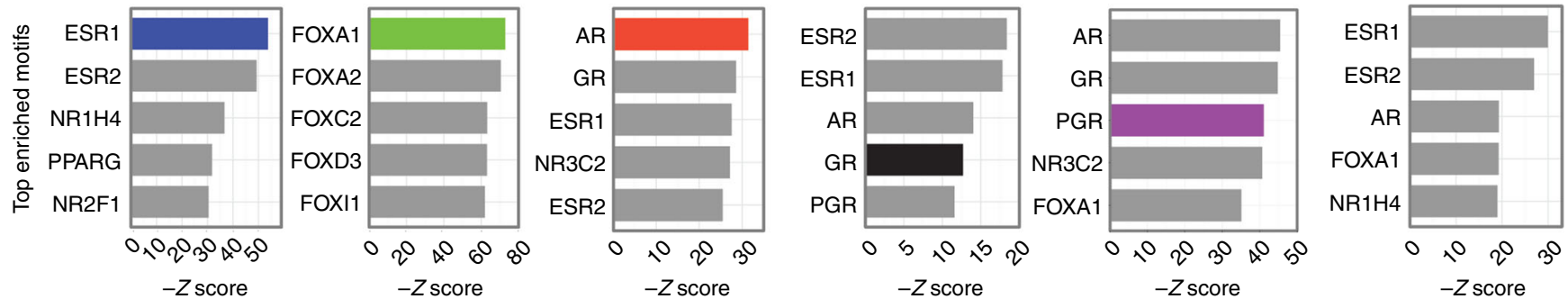
with ER $\alpha$ (Fig. 2c), which was also seen in female breast tumor and MCF7 cells (Supplementary Fig. 6A, B).

$\mathrm{MBC}$ is $\mathrm{ER} \alpha$-driven ${ }^{37,38}$. Therefore, a one-to-one comparison of binding sites of $\mathrm{ER} \alpha$ with each factor was performed (Fig. 2c, d and Supplementary Fig. 7), where only samples were analyzed in which both ER $\alpha$ and the other factor were profiled (peak selection as stated above and in figure legends). A significant proportion of $\mathrm{ER} \alpha$ binding sites overlap with $\mathrm{AR}$ and GR sites as seen in cell line data 39,40 which we confirmed in MBC (Fig. 2c, d). Interestingly, although $\mathrm{PR}$ modulates $\mathrm{ER} \alpha$ binding in $\mathrm{FBC}^{25}$, many $\mathrm{PR}$ sites in MBC were devoid of $\mathrm{ER} \alpha$ (46\%). These findings were confirmed in DNA binding correlation matrices (Fig. 2e). $\mathrm{ER} \alpha$ and $\mathrm{AR}$ show clustering within patient rather than between factors (Fig. 2e), indicating a stronger correlation between factors within the same tumor as compared to the same factor between tumors.

A dominant dogma of $\mathrm{ER} \alpha$ biology purports $\mathrm{ER} \alpha$ binding is dependent on its pioneering factor FOXA1, with $95 \%$ of binding found at enhancer regions ${ }^{11,29,30,41,42}$. In line with literature ${ }^{11}$, we find $\sim 50 \%$ overlap between ER $\alpha$ and FOXA1 in cell lines, where sites enriched for FOXA1 or ER $\alpha$ (Fig. 3a, b and Supplementary Fig. 8) were largely found in intronic and distal intergenic regions (Fig. 3c). These findings are in stark contrast to observations in both male and female breast cancers, in which ER $\alpha$ sites devoid of FOXA1 were strongly promoter-enriched (Fig. $3 a-c)$, suggesting the model systems currently used do not adequately capture the genomic distributions of $\mathrm{ER} \alpha$ found in clinical samples. In contrast to FOXA1-enriched sites, sites selectively occupied by $\mathrm{ER} \alpha$ were weaker for active enhancer mark H3K4me1 ${ }^{33}$ (Supplementary Fig. 9A, B). Interestingly, GATA3 was found at both the FOXA1-enriched and ER $\alpha$-enriched sites (Supplementary Fig. 9A). Motifs at ER $\alpha$ selective sites are related to ESR1 and devoid of forkhead motifs (Supplementary Fig. 10), which is in contrast to the total of ER $\alpha$ sites (Supplementary Fig. 5).

MBC subtypes differ in hormone receptor action. Having characterized $\mathrm{MBC}$ with respect to SHRs, GATA3 and FOXA1 DNA binding, we next performed gene expression analyses in these samples $(n=46)$. In order to assess underlying ER $\alpha$ binding patterns between published MBC intrinsic subtypes $\mathrm{M} 1$ and $\mathrm{M}_{2}{ }^{4}$, we first confirmed M1/M2 subtype clustering using our RNA-seq data set using only subtype genes (Fig. 4a). Supporting our hypothesis that ER $\alpha$ function may deviate between M1 and M2 subtypes, we found 1395 differential ER $\alpha$ DNA binding sites (Fig. 4b). Analogous analyses were not performed for other factors than $\mathrm{ER} \alpha$, since ChIP-seq datastreams were not sufficiently powered to represent both the M1 and M2 subtypes (Supplementary Fig. 1). With available datastreams, we confirmed the occupancy of FOXA1, AR, GR, PR, and GATA3 at these differential ER $\alpha$ DNA binding sites (Fig. 4c). M1- and M2-specific sites were comparable in genomic location and motif usage (Fig. 4d, e and Supplementary Fig. 11). Genes with proximal binding sites ( $<20 \mathrm{~kb}$ or within the gene body) were subsequently examined for molecular and biological associations using pathway analysis (IPA, Qiagen). Both ER $\alpha$ ChIP-seq associated M1/ M2 genes and gene expression-based M1/M2 genes strongly associated with ER $\alpha$ pathway indicators as expected, though some additional regulators are specifically found in the previously reported expression-based classification, such as ERBB2 and KRAS (Fig. 4f). Interestingly, among canonical pathways, AR signaling was the only hormonal signaling pathway more associated with the $\mathrm{ER} \alpha$ binding based genes compared to the gene expression-based genes (Fig. $4 \mathrm{~g}$ ), in line with the strong overlap of $\mathrm{AR} / \mathrm{ER} \alpha$ binding in these tumors (Figs. 2, 4c).

Comparing genomics of ER $\alpha$ and FOXA1 between genders. As $\mathrm{ER} \alpha$ is the key driver and therapeutic target in both genders, we compared ER $\alpha$ chromatin binding in female (17 from Ross-Innes et al. ${ }^{10}, 9$ from Jansen et al. ${ }^{9}$ and 10 generated in-house) and male (30 generated in-house) breast tumors (Fig. 5a), along with its pioneer factor FOXA1 ( $n=7$ for both genders) (Fig. 5b). Interestingly, no clear differences in $\mathrm{ER} \alpha$ and FOXA1 binding was found between genders, on the level of peak overlap ratio (Fig. 5a-d) or relative read counts in peaks (Fig. 5e, f). For ER $\alpha$ and FOXA1 sites found in $\geq 50 \%$ of male tumors, signal was observed in female samples at comparable intensity (Fig. 5e, f, Supplementary Fig. 12A, B, Supplementary Fig. 13), and vice versa. Furthermore, motif enrichment at ER $\alpha$ and FOXA1 sites was highly comparable between genders (Fig. 5g, h). While clear clustering was observed for ER $\alpha$ between (male and female) tumors and cell lines (Fig. 5a), no separation on gender was observed for any of the factors studied in an integrative analysis (Supplementary Fig. 14), as well as separately for all factors studied (Fig. 5a, b, Supplementary Fig. 15). ER $\alpha$ sites that classify FBC on outcome ${ }^{10}$ were used to predict male outcome ( $k$-nearest neighbor classifier; Methods section), and a weak but similar trend of $\mathrm{ER} \alpha$ signal strength was observed in these sites (Fig. 5i). However, overall survival (OS) was not significantly different between the two groups of male patients (Supplementary Fig. 16). These results suggest that although the vast majority of $\mathrm{ER} \alpha$ and FOXA1 sites are conserved between breast cancers from both genders, $\mathrm{ER} \alpha$ sites indicative for outcome in FBC may not be applicable in the male disease.

Genomic profiles of ER $\alpha$ and FOXA1 stratify MBC patients on outcome. Since prognostic ER $\alpha$ sites in female tumors do not seem to be indicative for male patient outcome in our MBC series, we analyzed binding sites of $\mathrm{ER} \alpha$ and pioneer factor FOXA1 in $\mathrm{MBC}$ for outcome prediction. Analogous analyses for $\mathrm{ER} \alpha /$ GATA3 were not performed due to insufficient power due to

Fig. 1 Study schematic and steroid hormone receptor binding in male breast cancer. a A graphic visualization of the study design. The male silhouette was from Wikipedia (https://en.wikipedia.org/wiki/File:Male_Bathroom_Symbol.png). The female silhouette was from Wikipedia (https://commons. wikimedia.org/wiki/File:Toilet_women.svg), from a collection commissioned by the United States Department of Transportation, designed by AIGA http:// www.aiga.org/content.cfm/symbolsigns, and converted into SVG by Wikipedia-user Lateiner. The image is used under a CC-BY 2.5 license. b Genome browser snapshot, depicting two known ER $\alpha$ bound regions with read counts for 2 random samples chosen for each factor. Genomic coordinates and read counts are indicated above. c Heatmaps depicting peak intensity in primary tumors for 30 ER $\alpha$ (blue), 7 FOXA1 (light green), 10 AR (red), 7 GR (black), 4 PR (purple), and 3 GATA3 (dark green) binding events ( $\pm 5 \mathrm{~kb}$ from the peak center (triangle)). 5079 ER $\alpha$ sites were determined using the consensus ER $\alpha$ binding sites identified in at least $50 \%$ of patients ( 15 out of 30 samples). d Pie charts depicting genomic distributions for the consensus binding sites of each of the factors: AR (shared in 5 out of 10 samples), FOXA1 ( 3 out of 7 samples), PR ( 2 out of 4 samples), GR ( 3 out of 7 samples), and GATA3 (1 out of 3 samples). e Negative Z-score of the top 5 sequence motifs for binding sites of each factor depicted as a barplot. Colored (non-gray) bar represents the target factor's sequence motif 
a

ChIP-seq peak overlaps

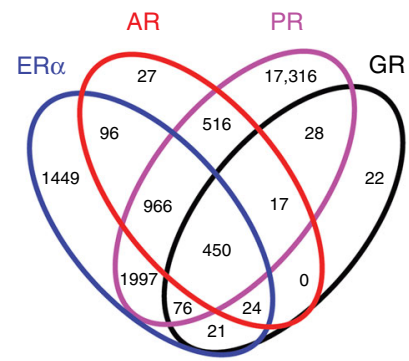

-ER $\alpha=F O X A 1=G A T A 3$

$\because \mathrm{AR}\|\mathrm{GR}\| \mathrm{PR}$ b

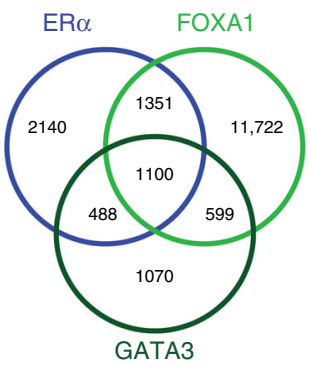

ChIP-seq peak correlation matrix

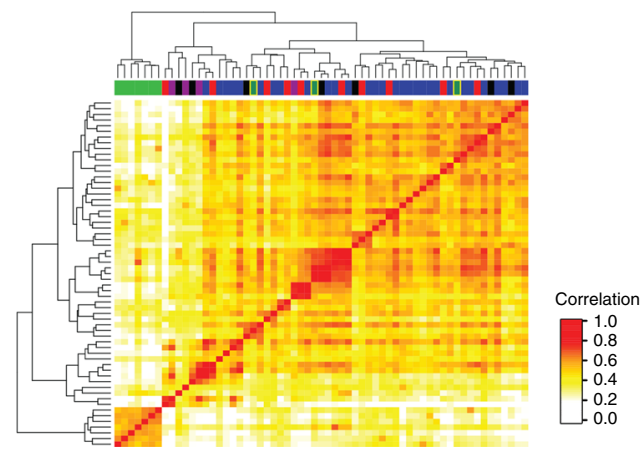

C Pair-wise peak overlap
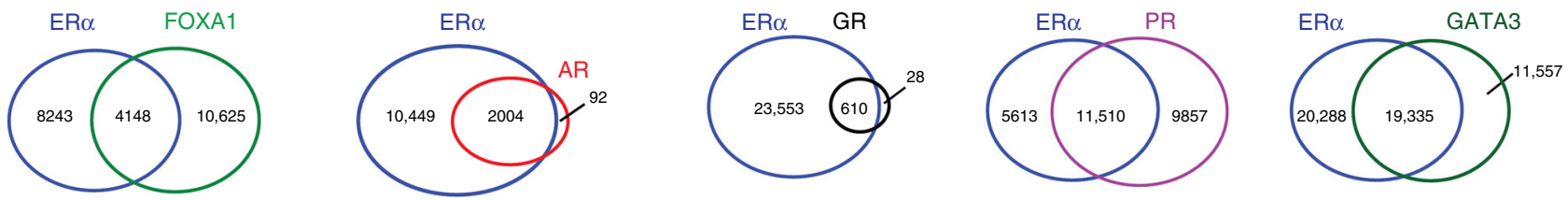

d Pair-wise ChIP-seq
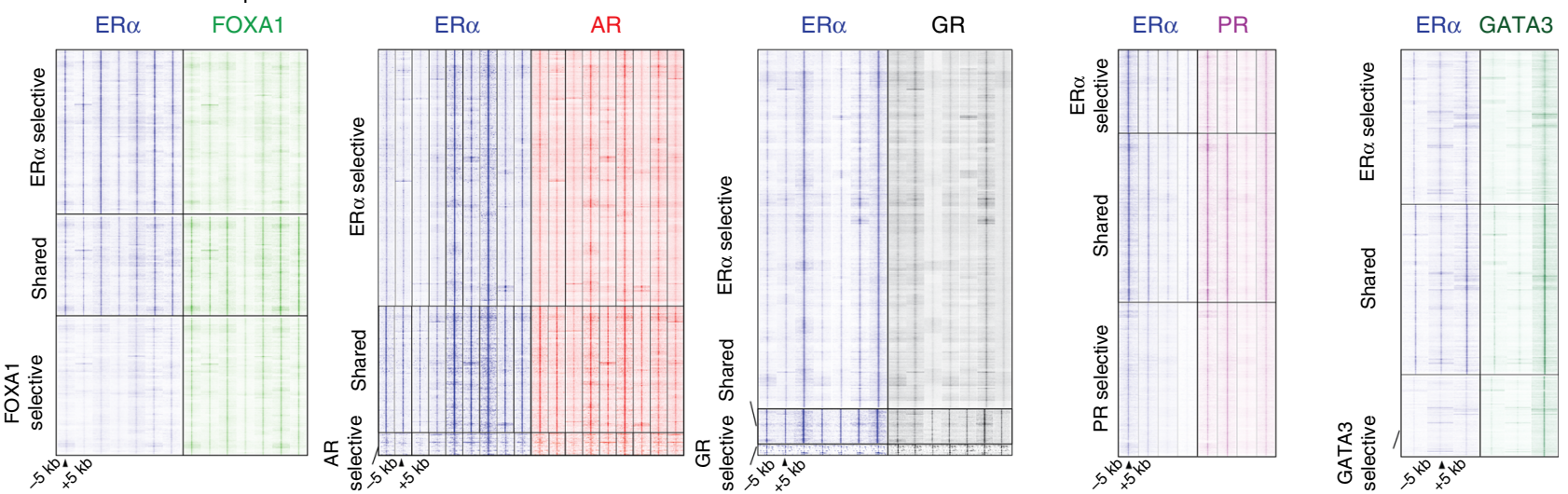

\section{e Pair-wise correlation matrices}
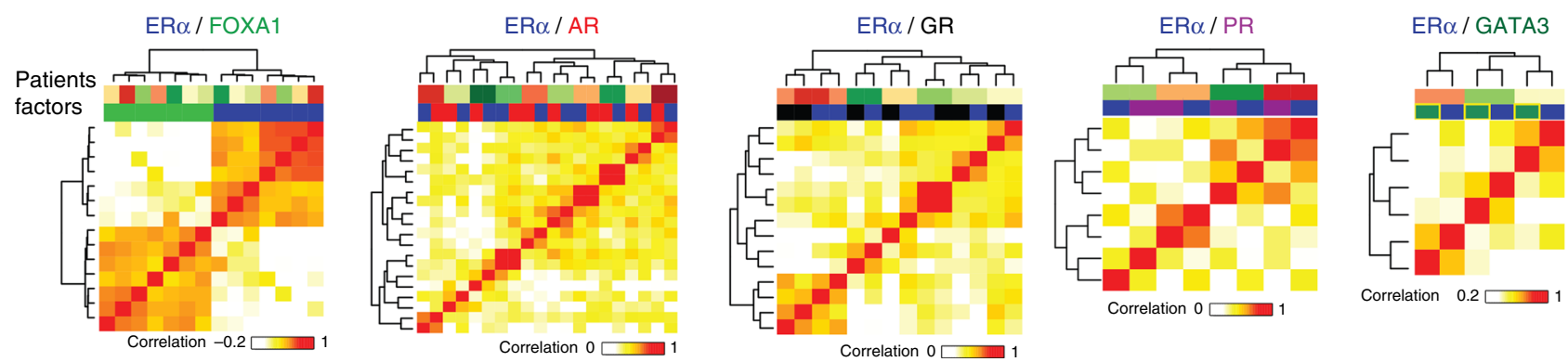

Fig. 2 Multifactorial ChIP-seq data integration. a Venn diagram depicting the overlap of ER $\alpha$ with steroid hormone receptors (left) and overlap of ER $\alpha$ with FOXA1 and GATA3 (right). b Unsupervised clustering correlation matrix of bound regions among all factors. Top bar indicates the factors: ER $\alpha$ (blue), AR (red), PR (purple), GR (black), FOXA1 (light green), and GATA3 (dark green/orange border). Pearson correlation is depicted from 0 (white) to 1 (dark green). Sites used to construct the matrix are the union of all consensus binding sites of the factors. c Individual Venn diagrams depicting the pairwise overlap of ER $\alpha$ with each factor. Consensus binding sites for each factor were used (Fig. 1d), and overlap with ER $\alpha$ sites from the same tumors was assessed, taking the same threshold. d Heatmaps indicating the binding peak intensity for each combination in (c). In each panel, ER $\alpha$ (left) and factor (e.g., FOXA1, right) ChIP-seq data are depicted. Shown are peaks selectively observed for ER $\alpha$ (top), selectively observed for other factors (bottom), or shared between them (middle). e Correlation heatmap for ChIP-seq data sets between ER $\alpha$ and the other factors. Colors in top bar indicate patient (top) and factor (bottom) 
a ChIP-seq in ER $\alpha$ or FOXA1 enriched sites across biological systems

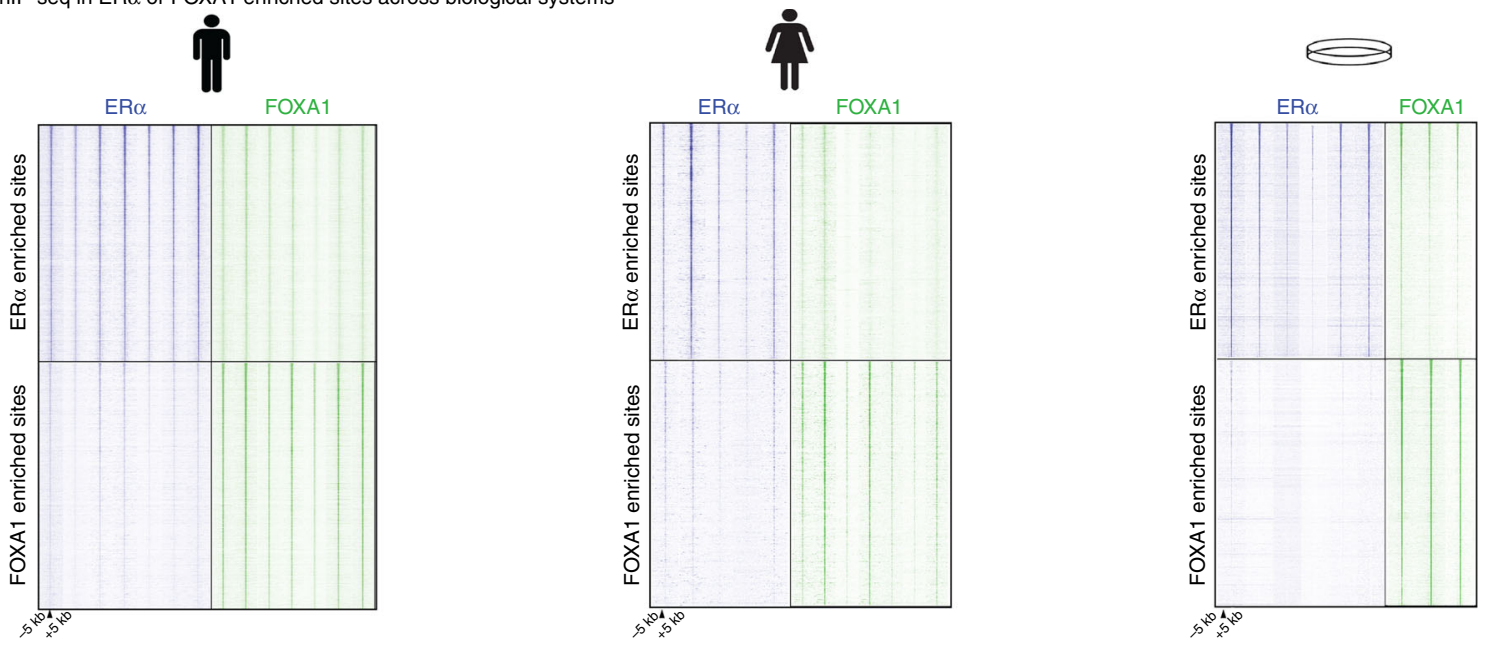

b ChIP-seq extra snapshots in ER $\alpha$ or FOXA1 enriched sites across biological systems
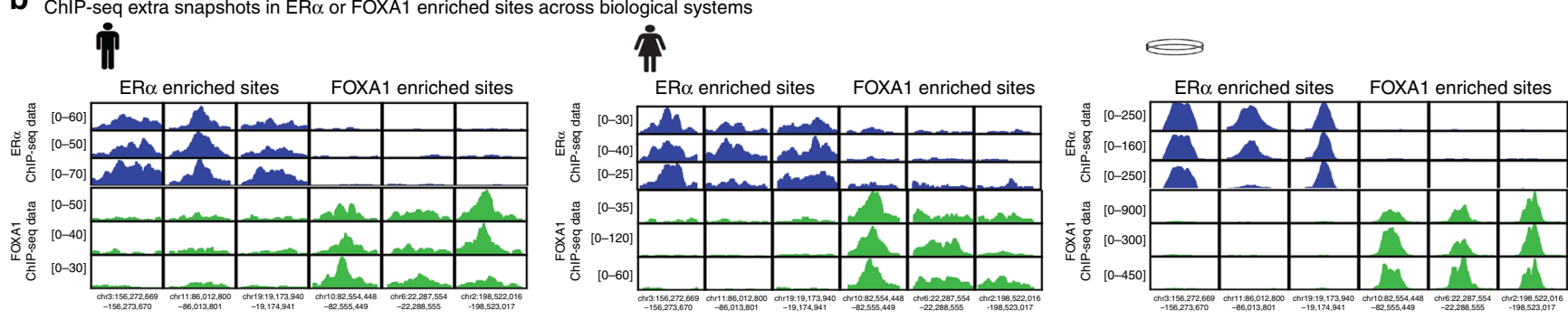

C Genomic distributions of ER $\alpha$ or FOXA1 enriched sites across biological systems
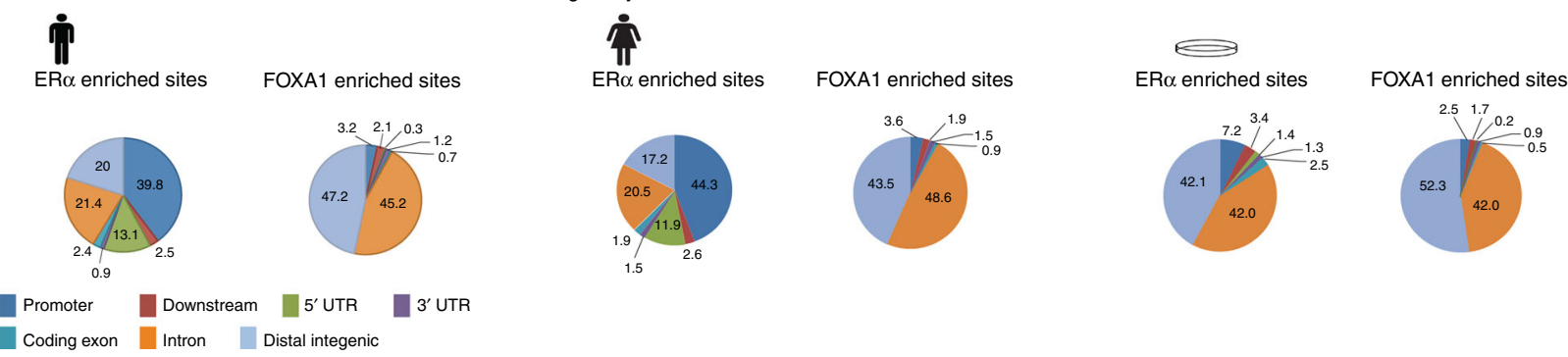

Fig. $3 \mathrm{ER} \alpha$ - and FOXA1-enriched binding sites across biological systems. a Heatmaps indicating the binding peak intensity in sites, differentially enriched between ER $\alpha$ (blue) and FOXA1 (green). Left, middle and right columns indicate male tumor, female tumor and female cell line data, respectively. The male silhouette was from Wikipedia (https://en.wikipedia.org/wiki/File:Male_Bathroom_Symbol.png). The female silhouette was from Wikipedia (https:// commons.wikimedia.org/wiki/File:Toilet_women.svg), from a collection commissioned by the United States Department of Transportation, designed by AIGA http://www.aiga.org/content.cfm/symbolsigns, and converted into SVG by Wikipedia-user Lateiner. The image is used under a CC-BY 2.5 license. $\mathbf{b}$ Genome browser snapshot depicting sites differentially bound by ER $\alpha$ or FOXA1 across biological systems. Genomic coordinates and read count are indicated. c Pie charts indicating genomic distribution of ER $\alpha$ - (left) and FOXA1-enriched (right) sites across biological systems

small $(n=3)$ sample size. Based on lymph node status, indicative for overall survival (Supplementary Fig. 17), $365 \mathrm{ER} \alpha$ and 470 FOXA1 sites differed (Fig. 6a). Differential ER $\alpha$ and FOXA1 sites between patient subgroups were coupled to proximal genes $(<20 \mathrm{~kb}$ or within the gene body). Unsupervised hierarchical clustering revealed clusters dominated by either of M1 or M2 subtypes, both in our cohort (Fig. 6b) and a validation set ${ }^{4}$ (Fig. 6c; $n=66$ patients). We performed logistic regression with elastic net regularization ${ }^{43}$ to construct a supervised binary classification model by which predictive gene signatures could be identified, which was trained in our cohort and tested in the validation cohort. Both ER $\alpha$ - and FOXA1-based classifiers captured predictive features, which were outperformed by the union of both classifier gene lists (Fig. 6d, e). A bootstrapping analysis confirmed that comparable performance is rarely achieved with random gene sets $(p=0.013$, one-tailed test with bootstrapped performance distribution; Methods section; Supplementary Fig. 18). Dividing patients into two groups of equal size based on the signature (high-risk and row-risk group of LN-status) significantly classified patients on distant metastasis free survival (DMFS; $p=0.048$, log-rank test; Fig. 6f; Methods section), which was marginally significant in a multivariate Cox analysis including LN-status ( $p=0.066$, Cox proportional hazards test). The gene expression signature is a linear combination of gene expression levels, in which 14 contributing genes classify MBC on outcome (Fig. 6g). Cumulatively, we show that global ER $\alpha$ and FOXA1 chromatin binding selectivity reveals gender-specific prognostic features that successfully classify MBC patients on survival.

\section{Discussion}

This work has characterized the DNA binding landscape of ER $\alpha$ in male breast cancer, along with its pioneer factors FOXA1, 
GATA3, and enhancer-enriched histone modification H3K4me1. In addition, we present the first set of DNA binding data in breast tumor specimens for other members of the steroid hormone receptor family: AR, GR, and PR. Our findings have indicated that the majority of ER $\alpha$ binding sites in both male and female breast tumors are FOXA1-independent and are found at active promoter regions, indicating a novel and unexpected mode of $\mathrm{ER} \alpha$ function. These results are in stark contrast to cell line-based studies that illustrated the majority of $\mathrm{ER} \alpha$ sites shared with FOXA1, mainly found at enhancers ${ }^{11}$. Our findings highlight the a

Gene expression and M1/M2 status

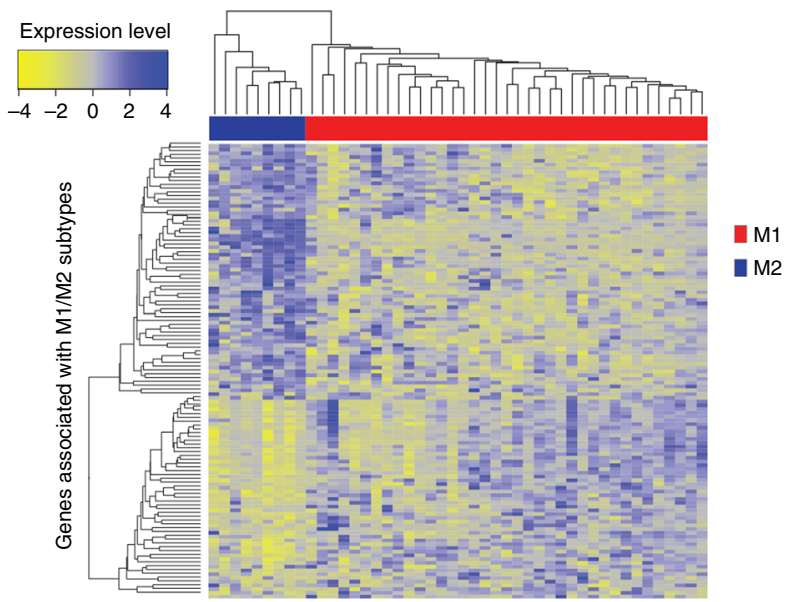

b

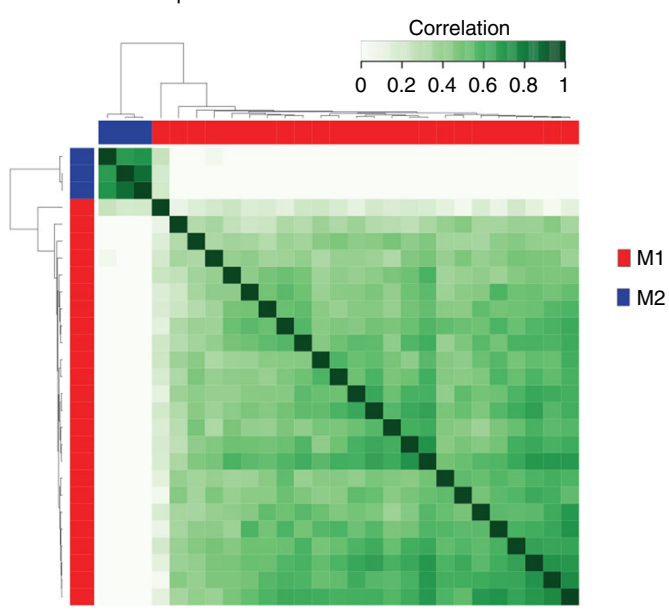

C

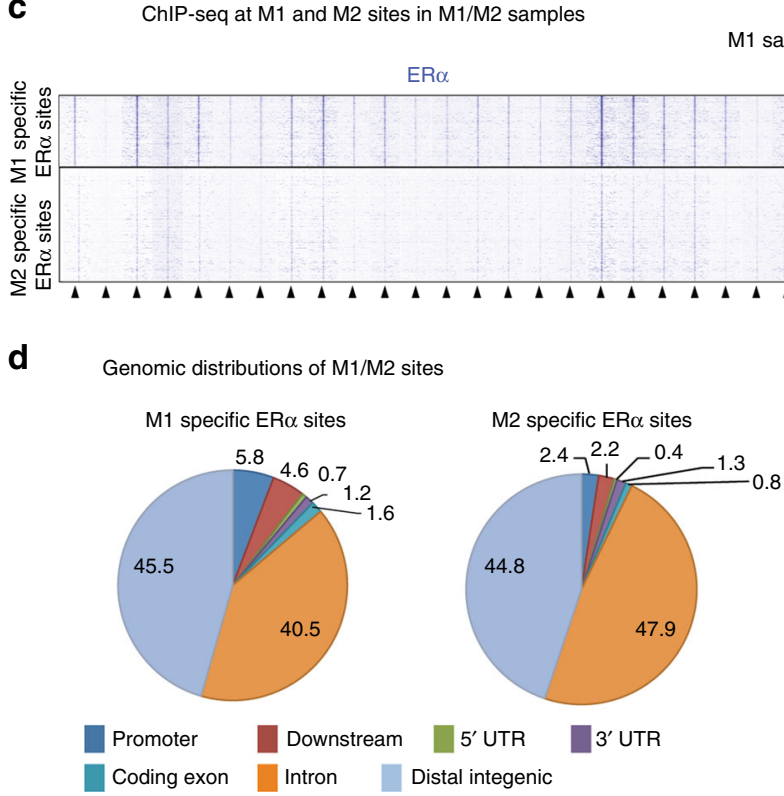

e

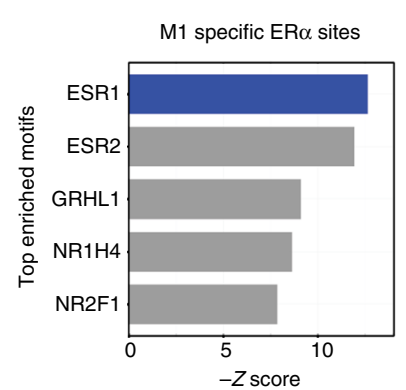

f

samples

M2 samples

FOXA1

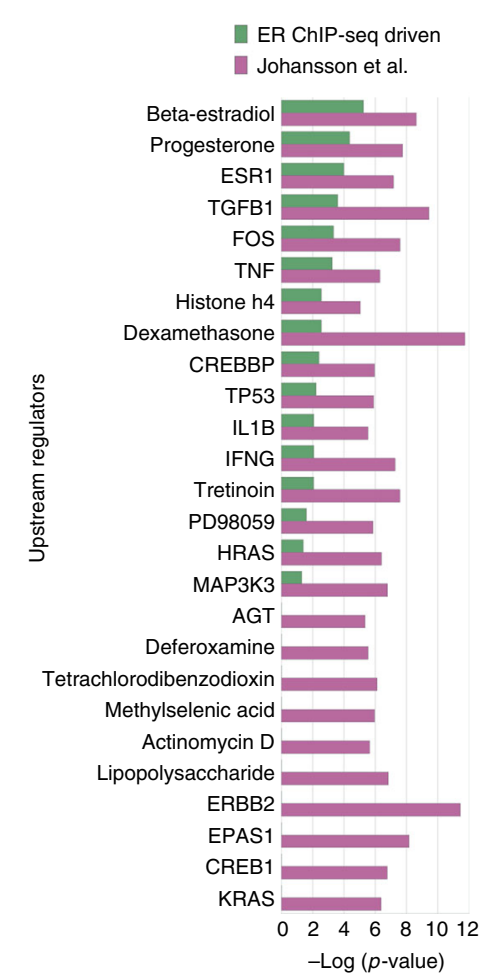

Molecular and biological processes

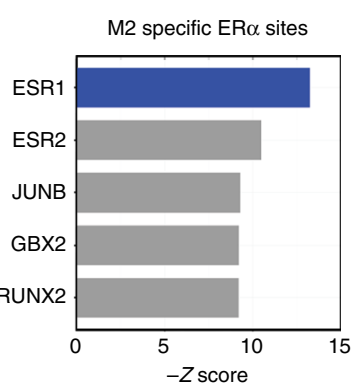

AR

GR GATA3

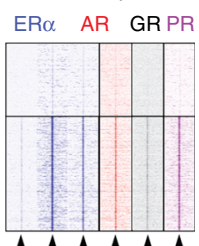

g

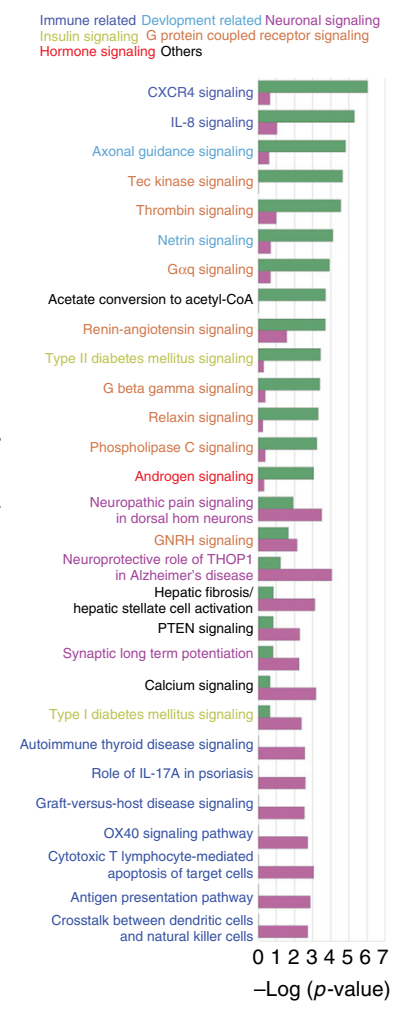


necessity to address transcription factor functioning in the physiological context of human tissue, rather than limiting analyses to cell line models.

Our data reveal that genomic functions of $\mathrm{ER} \alpha$ and $\mathrm{AR}$ in male breast tumors are largely overlapping, which strongly co-localized with GR and PR at the same regions. Even though many sites for GATA3, FOXA1, and PR were not shared with ERo, both AR and GR show virtually no unique binding sites with respect to ER $\alpha$ binding. AR has been shown to compensate for ER $\alpha$ in $\mathrm{ER} \alpha-/ \mathrm{AR}+$ female breast cancers ${ }^{40,44}$, however the biological interaction between $\mathrm{ER} \alpha$ and $\mathrm{AR}$ is relatively unknown in both $\mathrm{FBC}$ and $\mathrm{MBC}^{45}$. The observed genomic overlap of steroid hormone receptor binding profiles is likely due to the close sequence homology of DNA binding domains between all steroid hormone receptors, which warrants potential competition between them in DNA binding. Alternatively, genomic overlap of SHR binding profiles may be the consequence of 'tethering', in which factors associate to the DNA indirectly through complex formation with DNA-bound factors ${ }^{35}$, as was recently described for ER $\alpha$ and $\mathrm{PR}^{25}$. Such genomic convergence of steroid hormone receptor action in tumors may provide a novel starting point for pharmaceutical intervention strategies, yielding direct biological rationale for the use of small molecule therapeutics to target AR (e.g., clinicaltrials.gov NCT01990209), GR or PR in hormone receptor-positive breast cancer. As $\mathrm{MBC}$ is a rare cancer with limited numbers of available tumors for genomic studies, ChIPseq analyses for some factors including PR and GATA3 were performed on relatively low number of tumors. To focus our analyses on the most-robust peaks and thus minimizing potential impact of patient heterogeneity, for all SHRs we only considered peaks that were found in around $50 \%$ of patient samples. This could be considered a rather conservative approach compared to other tissue-derived ChIP-seq papers, where the union of all peaks ${ }^{46}$ or peaks identified in at least 2 out of 21 tumors $^{10}$ were used as consensus for analyses. Nonetheless, results for PR and GATA3 still warrant validation in larger cohorts.

Although, we found the vast majority of ER $\alpha$ sites to be shared between male and female breast cancers, ER $\alpha$ sites that are associated with patient outcome appeared gender-selective. In line with these results, genomic selectivity of combinatorial steroid hormone receptor action is associated with the genderspecific intrinsic MBC subtypes M1 and M2. While these data suggest $\mathrm{ER} \alpha$ function may be driving these subtypes, causality can only be illustrated when cell line models, organoids or patientderived xenografts are available for mechanistic studies. As the most clinically relevant observation, we have identified distinct genomic signatures of ER $\alpha$ action, which selectively and exclusively classifies MBC patients on outcome. With differential binding of ER $\alpha$ and FOXA1 as a guide, we developed a gene expression signature that is significantly associated with DMFS in MBC patients. The union of genes under differential control of ER $\alpha$ and FOXA1 jointly classify patients on outcome, and it remains to be determined which transcription factor is facilitated by FOXA1 at these sites. The MLL3 histone methyltransferase may represent one candidate to be tested in future studies based on the published FOXA1 and MLL3 interaction in FBC cells ${ }^{47}$. The 14 genes classifier we identified may be of added value as male breast cancer-specific prognostic classifier, but further validation of these results would be needed. Furthermore, small molecule inhibitors are available for a number of the 14 genes represented in the classifier, such as CAMKK2 $\left(\right.$ STO-609) ${ }^{48}$, CAPN $9^{49}$, BACE2 $^{50}$, and TNFSF11 (aka RANKL $)^{51}$, and future studies could further elucidate whether these inhibitors may be applicable in the treatment of male breast cancer.

With this, we present the first comprehensive genomic overview of shared and unique features of four steroid hormone receptors in human cancer, with outcome prediction. By studying MBC, gender-selective features of ER $\alpha$ action were identified with potentially direct clinical implications, revealing the first biologydriven biomarker for outcome prediction in this highly understudied cancer-type.

\section{Methods}

Tumor specimens. In this study, primary male and female breast tumors were used, none of whom received neoadjuvant endocrine therapy. Male breast cancer patients received surgery at the Netherlands Cancer Institute-Antoni van Leeuwenhoek (NKI-AVL; Amsterdam, the Netherlands), University Medical Center Utrecht (UMCU; Utrecht, the Netherlands), Vrije Universiteit Medical Center (VUMC; Amsterdam, the Netherlands), Radboud University Medical Center (RadboudUMC; Nijmegen, the Netherlands), University Medical Center Groningen (UMCG; Groningen, The Netherlands), Leiden University Medical Center (LUMC; Leiden, the Netherlands), and Erasmus Medical Center (ErasmusMC; Rotterdam, the Netherlands).

Female breast cancer patients received surgery at the Netherlands Cancer Institute-Antoni van Leeuwenhoek (NKI-AVL; Amsterdam, the Netherlands). Tumor content and immunohistochemical analyses were assessed by pathological examination. For clinicopathological parameters, see Supplementary Tables 1 (male tumors) and 2 (female tumors). Local medical ethical authorities at abovementioned centers approved of the collection protocols. All samples were from anonymous left-over material, which would be discarded otherwise. Anonymized, coded leftover material which is not traced back to the patient and therefore does not interfere with care and/or prognosis, under strict requirements can be used without written informed consent according to Dutch legislation on Secondary $\mathrm{Use}^{52}$.

ChIP-seq and antibody validations. Tissue was processed as described previously ${ }^{53}$ with a few adaptations. In short, tissue was defrosted and crosslinked in solution A ( $50 \mathrm{mM}$ Hepes, $100 \mathrm{mM} \mathrm{NaCl}, 1 \mathrm{mM}$ EDTA, $0.5 \mathrm{mM}$ EGTA, pH=7.4) containing $2 \mathrm{mM}$ DSG, incubated for $25 \mathrm{~min}$ at room temperature while rotating. After 25 min formaldehyde was added to $1 \%$ final and incubated another $20 \mathrm{~min}$ at room temperature with rotation. Samples were quenched by adding a surplus of $0.2 \mathrm{M}$ glycine, pelleted by centrifugation ( $5^{\prime} @ 4000$ r.c.f. at $4^{\circ} \mathrm{C}$ ), washed with cold PBS and mechanically disrupted in cold PBS using a pellet pestle (Sigma). The PicoBioruptor (Diagenode) was used for sonication. For ChIP, antibodies were used to detect ER $\alpha$ (sc-543, Santa Cruz), AR (sc-816, Santa Cruz), FOXA1 (sc6554, Santa Cruz), PR (sc-7208, Santa Cruz), GR (12041 S lot 3, Cell Signaling Technology), GATA3 (sc-268, Santa Cruz), and H3K4me1 (ab8895, AbCam). Immunoprecipitated DNA was prepared for Illumina multiplex-sequencing with 10 samples per lane at 65 bp single end. For steroid hormone receptor ChIPs except $\mathrm{GR}, 5 \mu \mathrm{g}$ of antibody and $50 \mu \mathrm{l}$ dynabeads (Invitrogen) were used, for GR $7.5 \mu \mathrm{g}$ of antibody and $75 \mu \mathrm{l}$ dynabeads were used, and for FOXA1 and H3K4me1, $4 \mu \mathrm{g}$ of antibody and $40 \mu \mathrm{l}$ magnetic beads were used. ChIP-QPCR was performed to validate ChIP-seq data for ER $\alpha, \mathrm{GR}, \mathrm{AR}$, and FOXA1. For QPCR, relative enrichment of the RARA enhancer (chr:1738478661-38478809) (primers: $5^{\prime}$ GCTGGGTCCTCTGGCTGTTC-3' (FWD) and 5'

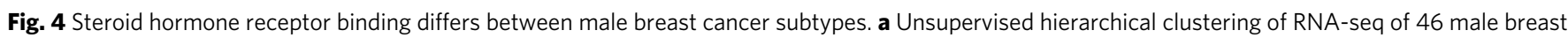
tumors, using the M1/M2 classifier genes defined by Johansson et al. ${ }^{4}$ Standardized gene expression value is denoted as the row Z-score and plotted in high expression (blue) and low expression (yellow) scale. Top row indicates M1 (red) or M2 (blue) classification. b Correlation plots of significantly differentially bound regions for ER $\alpha$ between $24 \mathrm{M} 1$ (red) and $3 \mathrm{M} 2$ (blue) tumors. Pearson correlation is depicted from 0 (white) to 1 (dark green) for all panels. c Heatmaps for all factors with known M1/M2 subtype classification, depicting raw signal intensities at differential bound ER $\alpha$ sites between $M 1$ and M2. d Genomic distribution for differentially ER $\alpha$-bound sites by $M 1$ and M2 subtypes. e Bar plots indicating top 5 sequence motifs enriched in M1 and M2 differentially ER $\alpha$-bound sites. $\mathbf{f}$ Ingenuity Pathway Analysis of Upstream Regulators and their $p$-values identified in both ER $\alpha$ binding site associated and Johannson et al. ${ }^{4}$ gene expression based driven M1/M2 genes. $\mathbf{g}$ Ingenuity Pathway Analysis of Canonical Pathways and their $p$-values identified in both $\mathrm{ER} \alpha$ binding site associated and Johannson et al. ${ }^{4}$ gene expression based driven M1/M2 genes 
a

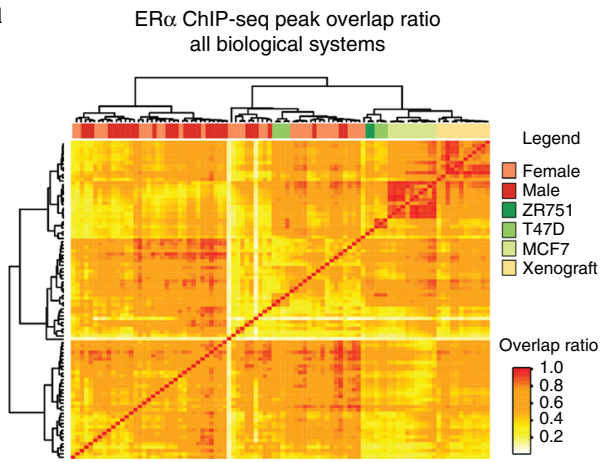

b

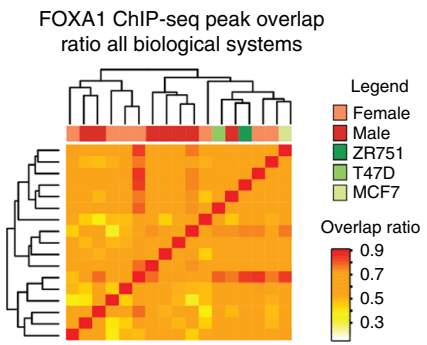

g

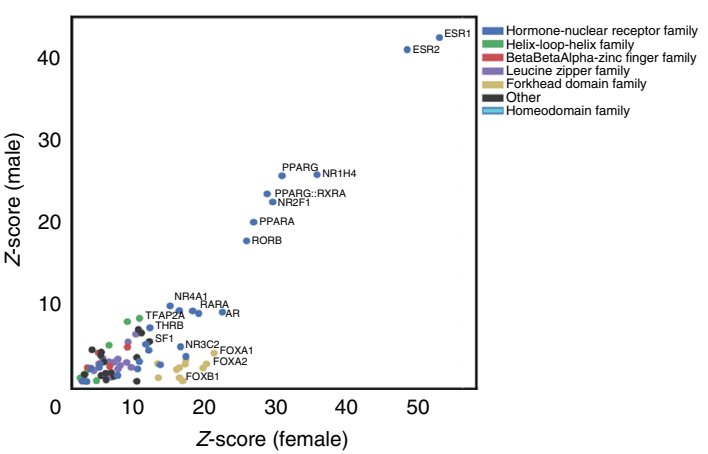

C

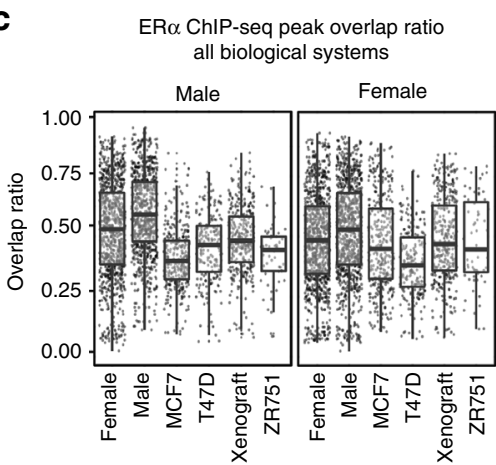

d

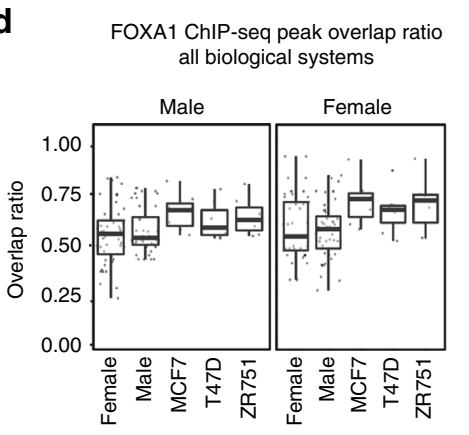

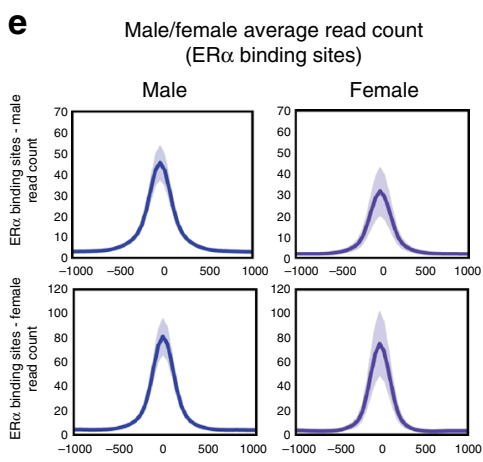

$\mathbf{f}$

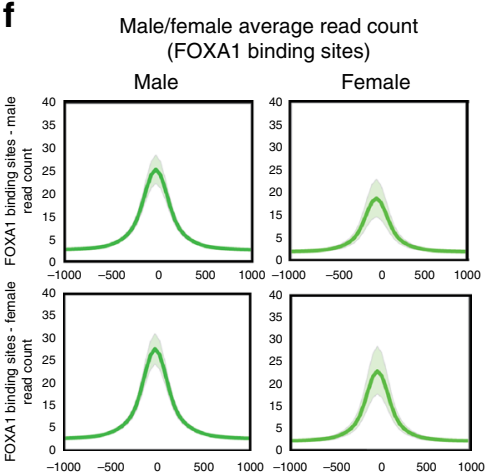

h

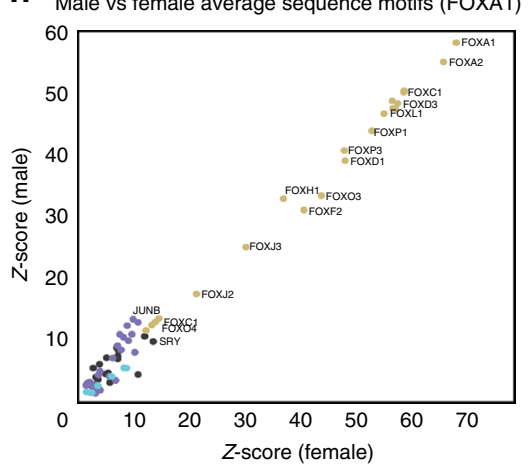

i
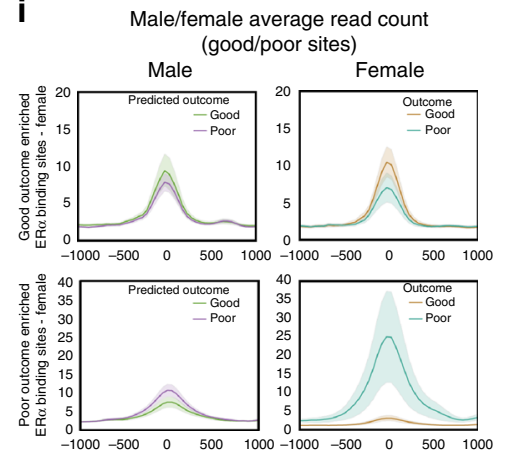

Fig. 5 Strong conservation of ER $\alpha$ and FOXA1 binding between genders. a Correlation plot of ER $\alpha$ bound regions across all biological systems. Top row indicates female tumors (salmon), male tumors (red), and female cell lines ZR751 (dark green), T47D (green), MCF7 (light green) and MCF7 xenograft (yellow) classification. Overlap ratio is depicted from 0 (white) to 1 (red). b Boxplots depicting all overlap values for ER $\alpha$ bound regions in male (left) and female (right) samples. c Correlation plot of FOXA1 bound regions across all biological systems. Top row indicates female tumors (salmon), male tumors (red), and cell lines ZR751 (dark green), T47D (green) and MCF7 (light green) classification. Overlap ratio is depicted from 0.3 (white) to 1 (red). d Boxplots depicting all overlap values for FOXA1 bound regions in male (left) and female (right) samples. e Average ER $\alpha$ read count profiles for male ER $\alpha$ binding sites ( $50 \%$ consensus, top panel) and female ER $\alpha$ binding sites (50\% consensus, bottom panel) in male (left) and female (right) datasets. $75 \%$ confidence interval of read count profiles are indicated with shading. $\mathbf{f}$ Average FOXA1 read count profiles for male FOXA1 binding sites (50\% consensus, top panel) and female FOXA1 binding sites ( $50 \%$ consensus, bottom panel) in male (left) and female (right) data sets. $75 \%$ confidence interval of average profiles are indicated with shading. $\mathbf{g}$ Scatter plot depicting Z-scores of significantly enriched motifs at ER $\alpha$ binding sites in male ( $y$-axis) and female ( $x$-axis) tumors. $\mathbf{h}$ Scatter plot depicting Z-scores of significantly enriched motifs at FOXA1 binding sites in male ( $y$-axis) and female ( $x$-axis) tumors. $\mathbf{i}$ Average ER $\alpha$ read count profiles of male (left) and female (right) tumors at the differential ER $\alpha$ binding sites ( $\pm 5 \mathrm{~kb}$ from the peak center) that can discriminate female outcome (top-good outcome enriched; bottom-poor outcome enriched). Patients are grouped based on outcome where indicated color is used for each group. $75 \%$ confidence interval of average profiles are indicated with shading

CCGGGATAAAGCCACTCCAA-3' (REV)) over a negative control region (primers: 5'-TGCCACACACCAGTGACTTT-3' (FWD) and 5'-ACAGCCAGAAGCTCCAAAAA-3' (REV)) was normalized over input, and plotted against the $\log$ (count/million) measurement from the same region from the MQ20 filtered aligned ChIP-seq file. SHR antibody specificity was validated by immunohistochemistry on U2OS cells transiently transfected with any of the SHRs of interest (Supplementary Fig. 3A). Antibody used for FOXA1 ChIP-seq also detects FOXA $2^{54}$ but only FOXA1 is expressed in these tumors (Supplementary Fig. 3B). Specificity for the antibody used for H3K4mel ChIP-seq $(\mathrm{ab} 8895)^{55,56}$ and GATA3 (sc-268) has been validated by others ${ }^{57,58}$. Publically available ChIP-seq data sets used are listed in Supplementary Table 3.

Immunohistochemistry. Immunohistochemistry staining for ER, PR, and AR was performed as previously described ${ }^{8}$. Immunohistochemistry of other factors was performed on a BenchMark Ultra autostainer (GATA3 and FOXA1) or Discovery Ultra autostainer (GR). Briefly, paraffin sections were cut at $3 \mu \mathrm{m}$, heated and deparaffinized in the instrument with EZ prep solution (Ventana Medical Systems). FOXA1 was detected using clone 2F83 (1/100,000 dilution, 16 min at RT, Seven 

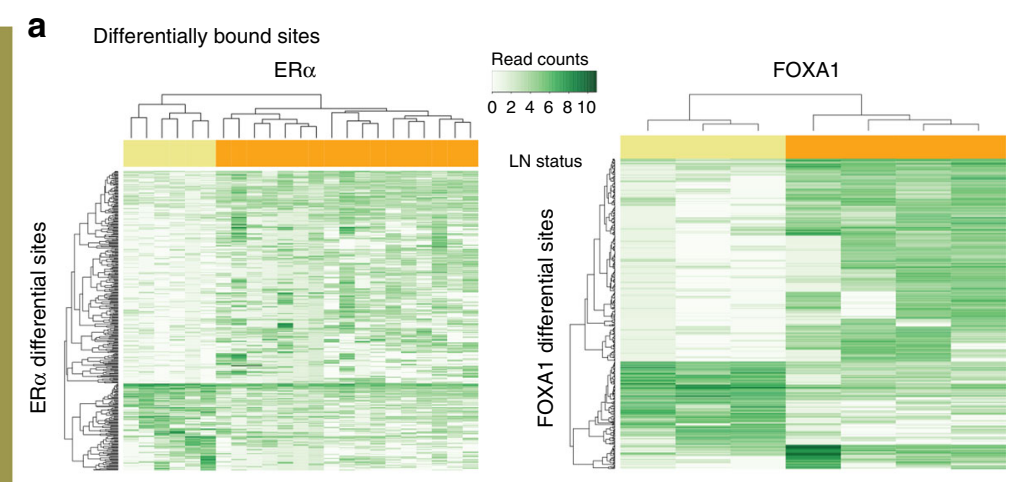

b Gene expression discovery cohort
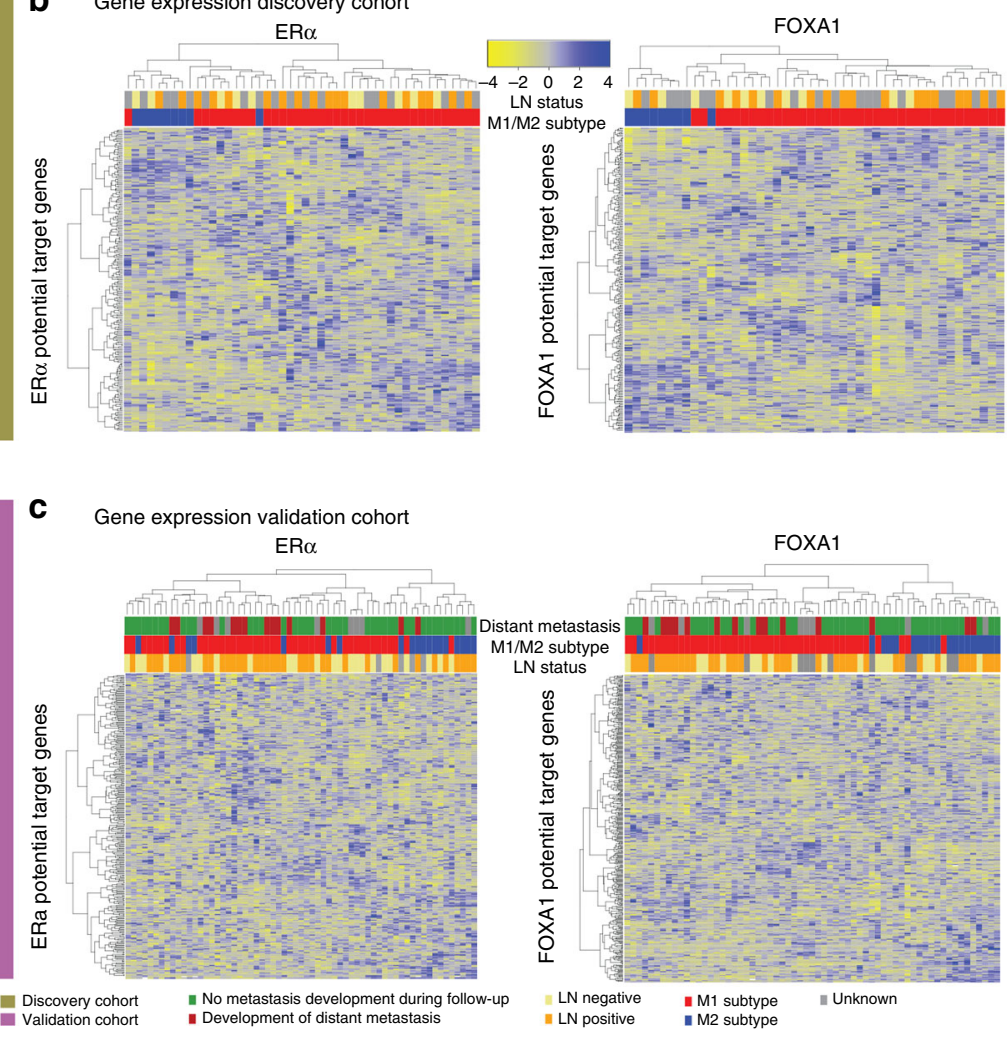

d
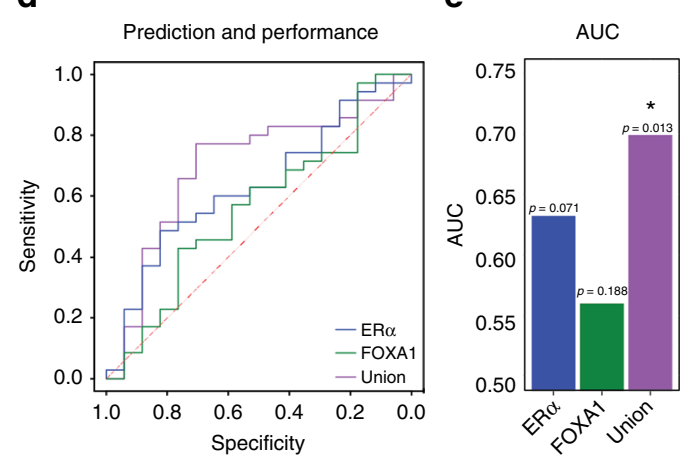

f

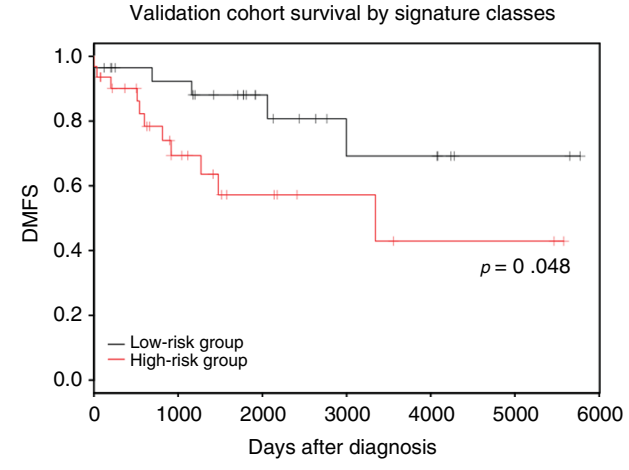

g

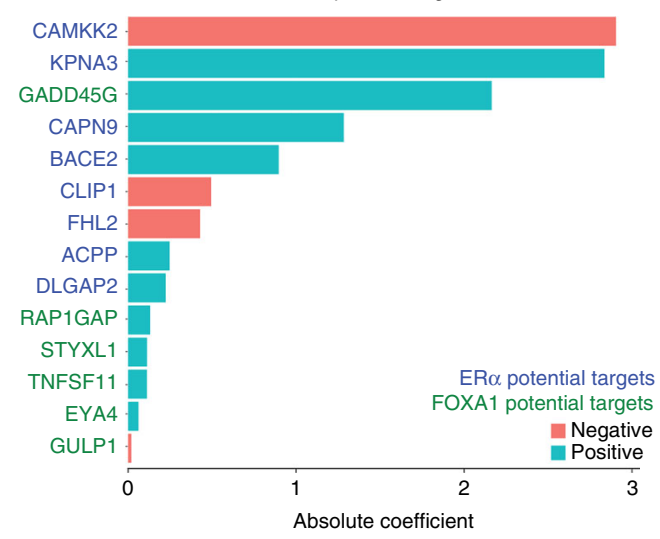

Fig. 6 Genomic selectivity of ER $\alpha / F O X A 1$ action classifies male breast cancers on outcome. a Unsupervised hierarchical clustering of ER $\alpha$ and FOXA1 peak intensities at sites that classify on negative (yellow) or positive (orange) LN-status. b Unsupervised hierarchical clustering of the potential target genes driven from ER $\alpha$ and FOXA1 binding sites that classify on LN-status (discovery cohort). Negative (yellow) and positive (orange) LN-status, M1 (red), and M2 (blue) subtypes are indicated by the color bars above. $\mathbf{c}$ The same analysis as in $\mathbf{b}$, using the validation cohort. Negative (yellow) and positive (orange) LN-status, M1 (red) and M2 (blue) subtypes as well as development of distant metastasis (red and green) are indicated by the color bars above.

d Receiver-operator characteristic (ROC) curve indicating predictive performance of binary classification models trained with potential target genes of ER $\alpha$ (blue), FOXA1 (green) or the union of the two (purple). e Area under curve of the ROC curves of $\mathbf{d}$, with $p$-values from bootstrapping analysis indicated on the top of each bar. $\mathbf{f}$ Kaplan-Meier analysis of two groups of patients (high- and low-risk groups in red and black lines, respectively) driven from the classification model trained with union of potential targets. Difference in survival is assessed with log rank test $(p$-value $=0.048)$. $\mathbf{g}$ Bar plot indicating 14 genes contributing to the classification model and their coefficients in the model. Predicted upstream regulator (blue - ER $\alpha$ and green - FOXA1) and sign of the coefficients (red - negative, green - positive) are indicated

Hills) followed by the UltraView Universal DAB Detection Kit (Ventana Medical Systems). GR was detected using clone D6H2L $\left(1 / 600\right.$ dilution, $1 \mathrm{~h}$ at $37^{\circ} \mathrm{C}$, Cell Signaling) and visualized using Anti-Rabbit HQ (Ventana Medical systems) for $12 \mathrm{~min}$ at $37^{\circ} \mathrm{C}$ followed by Anti-HQ HRP (Ventana Medical systems) for $12 \mathrm{~min}$ at $37^{\circ} \mathrm{C}$, followed by the ChromoMap DAB detection kit (Ventana Medical Systems). GATA3 was visualized using the OptiView DAB Detection Kit (Ventana Medical Systems). Slides were counterstained with Hematoxylin II and Bluing Reagent (Ventana Medical Systems). In the non-clinical setting (GR, FOXA1, GATA3), all scoring was performed on whole slides by a single pathologist (PJvD), blinded to patient status. In the clinical setting (ER, PR, and AR, we obtained positive/negative status from the clinical records. In accordance with clinical guidelines in the United States ${ }^{59}$, all samples were considered positive when at least $1 \%$ of nuclei were stained.

ChIP-seq bioinformatics. Sequenced samples were aligned to the reference human genome (Ensembl 37) using Burrows-Wheeler Aligner (BWA, v0.7.5a) with a mapping quality $>20$. Peak calling was performed using MACS (v1.4 $)^{60}$ and DFilter (v1.5) ${ }^{61}$, where only peaks were considered that were shared by the two peak callers. Genome browser snapshots were generated using IGV (v2.3.67), heatmaps were compiled using SeqMiner (v1.3.3 $)^{62}$ and genomic region 
enrichment analysis was performed with CEAS (v1.0.2) ${ }^{63}$. To generate consensus peaksets for each factor we used DiffBind (v1.14 $)^{10}$ with a cutoff defined where a peak must be seen in at least $50 \%$ of the samples for that factor. In the event that there were only 2 samples sequenced for a factor, only peaks were considered found in both samples. A list of the union of these sites was generated for Fig. 2a, b). For two-way Venn diagrams, we used these cutoffs in each factor in the set of 4 (ER $\alpha$ vs $\mathrm{AR}$ ), 3 (ER $\alpha$ vs FOXA1), and 2 (ER $\alpha$ vs GR/PR) (Fig. 2c, d). For differential binding analyses (Fig. 2e), DiffBind was used ${ }^{10}$ with the following parameters, FDR of 0.1 for comparison between two different factors and $p$-value of 0.01 for comparison between different LN-status. Given a set of binding site, genomic distribution and significantly enriched motifs were obtained using CEAS ${ }^{63}$ and SeqPos ${ }^{64}$ in Galaxy Cistrome (v1.0.0 $)^{64}$. All identified motifs were included in the wordcloud figures without removing close homologues, to prevent selection bias (Supplementary Figures 5, 10 and 11).

RNA isolation and RNA-seq. Sections ( $30 \mu \mathrm{m}$ thick) were cut from the frozen tumor tissues for RNA isolation. Total RNA was extracted using the mirVana miRNA isolation kit (Ambion, USA) according to the manufacturer's protocol until the end of F1. Quality and quantity of the total RNA was assessed by the 2100 Bioanalyzer (Agilent, USA). Total RNA samples having RIN $>8$ were subjected to library generation.

Strand-specific libraries were generated using the TruSeq Stranded mRNA sample preparation kit (Illumina, USA; RS-122-2101/2) according to the manufacturer's instructions (Illumina, Part \# 15031047 Rev. E). 3' adenylated and adapter ligated cDNA fragments were subject to 12 cycles of PCR. The libraries were analyzed on a 2100 Bioanalyzer using a 7500 chip (Agilent, Santa Clara, CA), diluted and pooled equimolarly into a multiplexed, $10 \mathrm{nM}$ sequencing pools and stored at $-20^{\circ} \mathrm{C}$. Strand-specific cDNA libraries were sequenced with 100 base paired-end reads on a HiSeq2500 using V4 chemistry (Illumina).

RNA-seq analysis. For all analyses we used the reference file Ensembl GRCh37.75. Adapter filtered reads were subject to STAR alignment (v2.4.2) ${ }^{65}$ carried out using default parameters. For expression analysis, HTSeq $(\mathrm{v} 0.6 .1 \mathrm{p} 1)^{66}$ was used to count reads for all genes in our RNA-seq samples using the htseq-count command. The DESeq2 (v1.16.0) $R$ package was then used to generate a gene expression matrix from these data ${ }^{67}$. Normalization of the data was carried out using the 'rlog' method within the package. Only samples with at least 5 reads across each gene were retained for further analyses $(N=46)$.

Prediction of male patient outcome on profiles of ER $\alpha$ and FOXA1. We constructed a $k$-nearest neighbor classifier based on ER $\alpha$ binding profile of female patients using scikit-learn module (v0.19) ${ }^{68}$ in Python. Taking read counts of ER $\alpha$ in the ER $\alpha$ binding sites that classify female outcome, male patient outcome is predicted by the outcome of five closest female data in terms of Minkowski distance

Logistic regression with elastic net regularization. We used $\mathrm{R}$ package glmnet $(\mathrm{v} 2.0)^{43}$ for constructing a logistic regression model using RNA-seq data in our cohort. Leave-one-out cross validation was performed for finding robust coefficients. Taking the independent validation cohort, linear combination of gene expression levels using the coefficients (gene expression signature) were obtained to rank the patients. Measuring sensitivity and specificity of LN-status prediction with varying threshold gives a receiver-operating characteristics (ROC) curve from which area under the curve (AUC) can be measured. A threshold dividing highand low-risk group was chosen as the median. We used potential target genes of $\mathrm{ER} \alpha$ and FOXA1, and union of potential targets to construct three classification models. Note that due to the high dimensionality, no contributing gene is found when the model is trained with whole genome data.

Bootstrapping analysis of classification model. We used the R package boot $(\mathrm{v} 1.3)^{69}$ for bootstrapping analysis. For each classification model, 1000 random models were constructed in the same manner but with random gene sets with the same size to obtain bootstrapped AUC distribution. Taking the bootstrapped AUC distribution as a null hypothesis distribution, significance of the performance was assessed by one-tailed test.

Survival analysis. We used R package Survival $(\mathrm{v} 2.38)^{70}$ for survival analysis. Given two patient groups based on data availability, the log-rank test was used for overall survival (OS) comparison in our cohort and distant metastasis free survival (DMFS) comparison in a validation cohort. Cox regression was used to assess association of LN-status and gene expression signature to survival. The available additional prognostic factors used in multivariate Cox regression were LN-status, endocrine therapy, chemotherapy, radiotherapy and age at diagnosis.

Data availability. All ChIP-seq data generated in the study are available on GEO repository: GSE104399 and RNA-seq data on GEO repository: GSE104730. All public data streams used in the study are listed in Supplementary Table 3.
Received: 30 November 2016 Accepted: 4 January 2018

Published online: 02 February 2018

\section{References}

1. Ferlay, J. et al. Cancer incidence and mortality worldwide: sources, methods and major patterns in GLOBOCAN 2012. Int. J. Cancer 136, E359-E386 (2015).

2. Ruddy, K. J. \& Winer, E. P. Male breast cancer: risk factors, biology, diagnosis, treatment, and survivorship. Ann. Oncol. 24, 1434-1443 (2013).

3. Rizzolo, P. et al. Male breast cancer: genetics, epigenetics, and ethical aspects. Ann. Oncol. 24(Suppl 8), viii75-viii82 (2013).

4. Johansson, I. et al. Gene expression profiling of primary male breast cancers reveals two unique subgroups and identifies $\mathrm{N}$-acetyltransferase-1 (NAT1) as a novel prognostic biomarker. Breast Cancer Res. 14, R31 (2012).

5. Ignatiadis, M. \& Sotiriou, C. Luminal breast cancer: from biology to treatment Nat. Rev. Clin. Oncol. 10, 494-506 (2013).

6. Droog, M., Beelen, K., Linn, S. \& Zwart, W. Tamoxifen resistance: from bench to bedside. Eur. J. Pharmacol. 717, 47-57 (2013).

7. Abreu, M. H. et al. Male breast cancer: looking for better prognostic subgroups. Breast 26, 18-24 (2016).

8. Severson, T. M. et al. Neoadjuvant tamoxifen synchronizes ER $\alpha$ binding and gene expression profiles related to outcome and proliferation. Oncotarget 7, 33901-33918 (2016)

9. Jansen, M. P. H. M. et al. Hallmarks of aromatase inhibitor drug resistance revealed by epigenetic profiling in breast cancer. Cancer Res. 73, 6632-6641 (2013).

10. Ross-Innes, C. S. et al. Differential oestrogen receptor binding is associated with clinical outcome in breast cancer. Nature 481, 389-393 (2012).

11. Hurtado, A., Holmes, K. A., Ross-Innes, C. S., Schmidt, D. \& Carroll, J. S. FOXA1 is a key determinant of estrogen receptor function and endocrine response. Nat. Genet. 43, 27-33 (2011).

12. Theodorou, V., Stark, R., Menon, S. \& Carroll, J. S. GATA3 acts upstream of FOXA1 in mediating ESR1 binding by shaping enhancer accessibility. Genome Res. 23, 12-22 (2012).

13. Isola, J. J. Immunohistochemical demonstration of androgen receptor in breast cancer and its relationship to other prognostic factors. J. Pathol. 170, 31-35 (1993)

14. Allegra, J. C. et al. Distribution, frequency, and quantitative analysis of estrogen, progesterone, androgen, and glucocorticoid receptors in human breast cancer. Cancer Res. 39, 1447-1454 (1979).

15. Chia, K., O'Brien, M., Brown, M. \& Lim, E. Targeting the androgen receptor in breast cancer. Curr. Oncol. Rep. 17, 4 (2015).

16. Wang, X., Yarid, N., McMahon, L., Yang, Q. \& Hicks, D. G. Expression of androgen receptor and its association with estrogen receptor and androgen receptor downstream proteins in normal/benign breast luminal epithelium. Appl. Immunohistochem. Mol. Morphol. 22, 498-504 (2014).

17. Castellano, I. et al. Androgen receptor expression is a significant prognostic factor in estrogen receptor positive breast cancers. Breast Cancer Res. Treat. 124, 607-617 (2010).

18. Wang, Q. et al. Androgen receptor regulates a distinct transcription program in androgen-independent prostate cancer. Cell 138, 245-256 (2009).

19. Xu, Y., Chen, S.-Y., Ross, K. N. \& Balk, S. P. Androgens induce prostate cancer cell proliferation through mammalian target of rapamycin activation and post-transcriptional increases in cyclin D proteins. Cancer Res. 66, 7783-7792 (2006).

20. Nevedomskaya, E. et al. Androgen receptor DNA binding and chromatin accessibility profiling in prostate cancer. Genom. Data 7, 124-126 (2016).

21. Stelloo, S. et al. Androgen receptor profiling predicts prostate cancer outcome. EMBO Mol. Med. 7, 1450-1464 (2015).

22. Sharma, N. L. et al. The androgen receptor induces a distinct transcriptional program in castration-resistant prostate cancer in man. Cancer Cell 23, 35-47 (2013).

23. Mohsin, S. K. et al. Progesterone receptor by immunohistochemistry and clinical outcome in breast cancer: a validation study. Mod. Pathol. 17, 1545-1554 (2004)

24. Shaaban, A. M. et al. A comparative biomarker study of 514 matched cases of male and female breast cancer reveals gender-specific biological differences. Breast Cancer Res. Treat. 133, 949-958 (2012).

25. Mohammed, H. et al. Progesterone receptor modulates ER $\alpha$ action in breast cancer. Nature 523, 313-317 (2015).

26. Abduljabbar, R. et al. Clinical and biological significance of glucocorticoid receptor (GR) expression in breast cancer. Breast Cancer Res. Treat. 150, 335-346 (2015).

27. Eeckhoute, J. et al. Positive cross-regulatory loop ties GATA-3 to estrogen receptor alpha expression in breast cancer. Cancer Res. 67, 6477-6483 (2007). 
28. Carroll, J. S. et al. Chromosome-wide mapping of estrogen receptor binding reveals long-range regulation requiring the forkhead protein FoxA1. Cell 122, 33-43 (2005).

29. Carroll, J. S. et al. Genome-wide analysis of estrogen receptor binding sites. Nat. Genet. 38, 1289-1297 (2006).

30. Lupien, M. et al. FoxA1 translates epigenetic signatures into enhancer-driven lineage-specific transcription. Cell 132, 958-970 (2008).

31. Zwart, W. et al. Oestrogen receptor-co-factor-chromatin specificity in the transcriptional regulation of breast cancer. EMBO J. 30, 4764-4776 (2011).

32. Kuilman, T. et al. CopywriteR: DNA copy number detection from off-target sequence data. Genome Biol. 16, 49 (2015).

33. Heintzman, N. D. et al. Distinct and predictive chromatin signatures of transcriptional promoters and enhancers in the human genome. Nat. Genet. 39, 311-318 (2007).

34. Curtis, C. et al. The genomic and transcriptomic architecture of 2,000 breast tumours reveals novel subgroups. Nature 486, 346-352 (2012).

35. Liu, Z. et al. Enhancer activation requires trans-recruitment of a mega transcription factor complex. Cell 159, 358-373 (2014).

36. Polman, J. A. E. et al. A genome-wide signature of glucocorticoid receptor binding in neuronal PC12 cells. BMC Neurosci. 13, 118 (2012).

37. Aisner, J., Ross, D. D. \& Wiernik, P. H. Tamoxifen in advanced male breast cancer. Arch. Intern. Med. 139, 480-481 (1979).

38. Doyen, J. et al. Aromatase inhibition in male breast cancer patients: biological and clinical implications. Ann. Oncol. 21, 1243-1245 (2010).

39. Karmakar, S., Jin, Y. \& Nagaich, A. K. Interaction of glucocorticoid receptor (GR) with estrogen receptor (ER) $\alpha$ and activator protein 1 (AP1) in dexamethasone-mediated interference of ER $\alpha$ activity. J. Biol. Chem. 288, 24020-24034 (2013).

40. Robinson, J. L. L. et al. Androgen receptor driven transcription in molecular apocrine breast cancer is mediated by FoxAl. EMBO J. 30, 3019-3027 (2011).

41. Cirillo, L. A. et al. Opening of compacted chromatin by early developmental transcription factors HNF3 (FoxA) and GATA-4. Mol. Cell 9, 279-289 (2002).

42. Laganière, J. et al. From the cover: location analysis of estrogen receptor alpha target promoters reveals that FOXA1 defines a domain of the estrogen response. Proc. Natl Acad. Sci. USA 102, 11651-11656 (2005).

43. Friedman, J., Hastie, T. \& Tibshirani, R. Regularization paths for generalized linear models via coordinate descent. J. Stat. Softw. 33, 1-22 (2010).

44. Doane, A. S. et al. An estrogen receptor-negative breast cancer subset characterized by a hormonally regulated transcriptional program and response to androgen. Oncogene 25, 3994-4008 (2006).

45. Severson, T. M. \& Zwart, W. A review of estrogen receptor/androgen receptor genomics in male breast cancer. Endocr. Relat. Cancer 24, R27-R34 (2017).

46. Kron, K. J. et al. TMPRSS2-ERG fusion co-opts master transcription factors and activates NOTCH signaling in primary prostate cancer. Nat. Genet. 49, 1336-1345 (2017).

47. Jozwik, K. M., Chernukhin, I., Serandour, A. A., Nagarajan, S. \& Carroll, J. S. FOXA1 directs H3K4 monomethylation at enhancers via recruitment of the methyltransferase MLL3. Cell Rep. 17, 2715-2723 (2016).

48. Hawley, S. A. et al. Calmodulin-dependent protein kinase kinase-beta is an alternative upstream kinase for AMP-activated protein kinase. Cell Metab. 2, 9-19 (2005).

49. Chen, C.-J., Nguyen, T. \& Shively, J. E. Role of calpain-9 and PKC-delta in the apoptotic mechanism of lumen formation in CEACAM1 transfected breast epithelial cells. Exp. Cell Res. 316, 638-648 (2010).

50. Canalis, E., McCarthy, T. L. \& Centrella, M. The role of growth factors in skeletal remodeling. Endocrinol. Metab. Clin. 18, 903-918 (1989).

51. Pageau, S. C. Denosumab. MAbs 1, 210-215 (2009).

52. Federation of Dutch Medical Scientific Scocieties (FDMSS). Dutch Medical Treatment Contracts Act and the Code of Conduct for Proper Secondary Use of Human Tissue of the Dutch Federation of Biomedical Scientific Societies. (FDMSS, 2011)

53. Zwart, W. et al. A carrier-assisted ChIP-seq method for estrogen receptorchromatin interactions from breast cancer core needle biopsy samples. $B M C$ Genom. 14, 232 (2013).

54. Droog, M. et al. Comparative cistromics reveals genomic cross-talk between FOXA1 and ER $\alpha$ in Tamoxifen-Associated Endometrial Carcinomas. Cancer Res. 76, 3773-3784 (2016).

55. Rothbart, S. B. et al. An interactive database for the assessment of histone antibody specificity. Mol. Cell 59, 502-511 (2015).

56. Egelhofer, T. A. et al. An assessment of histone-modification antibody quality. Nat. Struct. Mol. Biol. 18, 91-93 (2011).

57. Landt, S. G. et al. ChIP-seq guidelines and practices of the ENCODE and modENCODE consortia. Genome Res. 22, 1813-1831 (2012)

58. Witt, H. Encode DCC Antibody Validation Document. PDF validation document. Available at: https://www.encodeproject.org/antibodycharacterizations/2d3cfa05-cd0a-4a43-9f3e-2ec62260b17d/@@download/ attachment/human_GATA3_SC-268_validation_Farnham.pdf (2011).
59. Hammond, M. E. H. et al. American Society of Clinical Oncology/College Of American Pathologists guideline recommendations for immunohistochemical testing of estrogen and progesterone receptors in breast cancer. J. Clin. Oncol. 28, 2784-2795 (2010)

60. Zhang, Y. et al. Model-based analysis of ChIP-Seq (MACS). Genome Biol. 9, R137 (2008).

61. Kumar, V. et al. Uniform, optimal signal processing of mapped deepsequencing data. Nat. Biotechnol. 31, 615-622 (2013).

62. Ye, T. et al. seqMINER: an integrated ChIP-seq data interpretation platform. Nucleic Acids Res. 39, e35 (2011).

63. Shin, H., Liu, T., Manrai, A. K. \& Liu, X. S. CEAS: cis-regulatory element annotation system. Bioinformatics 25, 2605-2606 (2009).

64. Liu, T. et al. Cistrome: an integrative platform for transcriptional regulation studies. Genome Biol. 12, R83 (2011).

65. Dobin, A. et al. STAR: ultrafast universal RNA-seq aligner. Bioinformatics 29, 15-21 (2013)

66. Anders, S., Pyl, P. T. \& Huber, W. HTSeq--a Python framework to work with high-throughput sequencing data. Bioinforma. Oxf. Engl. 31, 166-169 (2015).

67. Love, M. I., Huber, W. \& Anders, S. Moderated estimation of fold change and dispersion for RNA-seq data with DESeq2. Genome Biol. 15, 550 (2014).

68. Pedregosa, F. et al. Scikit-learn: machine learning in Python. J. Mach. Learn. 12, 2825-2830 (2011).

69. Canty, A. \& Ripley, B. boot: Bootstrap R (S-Plus) Functions. R package version 1.3-20 (2016)

70. Therneau, T. A Package for Survival Analysis in S. version 2.38 (2015).

\section{Acknowledgements}

We thank all patients who contributed to this study. The authors would like to acknowledge all the members of hospitals that provided tumor material. Wilbert Zwart is supported by the Bas Mulder Award from the Dutch Cancer Society (KWF)/ALpe d'HuZes and a VIDI grant from the Netherlands Organization for Scientific Research (NWO). JWMM received funding through the Cancer Genomics Netherlands from The Netherlands Organization for Scientific research (NWO). The authors would also like to acknowledge the effort and support of The Netherlands Cancer Institute (NKI) Genomics Core Facility. In addition, we would like to acknowledge the NKI Core Facility Molecular Pathology and Biobanking (CFMPB) for supplying Biobank material and lab support, especially Ingrid Hofland for GR IHC optimization.

\section{Author contributions}

Experiments were performed by K.S., S.E.P.J., and W.Z. Computational analyses performed by T.M.S. and Y.K. Study supervision performed by P.v.d.G., P.J.v.D., S.C.L., L.W., and W.Z. Materials were provided by P.v.d.G., Q.F.M., J.W.M., C.H.M.v.D., E.B.,. I H., P.B., V.T.H.B.M.S., and P.J.v.D., and processed by N.D.t.H., C.B.M., and K.S. Manuscript was written by T.M.S., Y.K., and W.Z. with input from all other authors.

\section{Additional information}

Supplementary Information accompanies this paper at https://doi.org/10.1038/s41467 018-02856-2.

Competing interests: The authors declare no competing financial interests.

Reprints and permission information is available online at http://npg.nature.com/ reprintsandpermissions/

Publisher's note: Springer Nature remains neutral with regard to jurisdictional claims in published maps and institutional affiliations.

Open Access This article is licensed under a Creative Commons Attribution 4.0 International License, which permits use, sharing, adaptation, distribution and reproduction in any medium or format, as long as you give appropriate credit to the original author(s) and the source, provide a link to the Creative Commons license, and indicate if changes were made. The images or other third party material in this article are included in the article's Creative Commons license, unless indicated otherwise in a credit line to the material. If material is not included in the article's Creative Commons license and your intended use is not permitted by statutory regulation or exceeds the permitted use, you will need to obtain permission directly from the copyright holder. To view a copy of this license, visit http://creativecommons.org/ licenses/by/4.0/.

(c) The Author(s) 2018 